\title{
Fully coupled functional equations for the quark sector of QCD
}

\author{
Fei Gao®, ${ }^{1}$ Joannis Papavassiliou $\odot,{ }^{2}$ and Jan M. Pawlowski ${ }^{1,3}$ \\ ${ }^{1}$ Institut für Theoretische Physik, Universität Heidelberg, \\ Philosophenweg 16, 69120 Heidelberg, Germany \\ ${ }^{2}$ Department of Theoretical Physics and IFIC, University of Valencia and CSIC, E-46100 Valencia, Spain \\ ${ }^{3}$ ExtreMe Matter Institute EMMI, GSI, Planckstrasse 1, 64291 Darmstadt, Germany
}

(Received 1 March 2021; accepted 12 April 2021; published 14 May 2021)

\begin{abstract}
We present a comprehensive study of the quark sector of $2+1$ flavor QCD, based on a self-consistent treatment of the coupled system of Schwinger-Dyson equations for the quark propagator and the full quarkgluon vertex in the one-loop dressed approximation. The individual form factors of the quark-gluon vertex are expressed in a special tensor basis obtained from a set of gauge-invariant operators. The sole external ingredient used as input to our equations is the Landau gauge gluon propagator with $2+1$ dynamical quark flavors, obtained from studies with Schwinger-Dyson equations, the functional renormalization group approach, and large volume lattice simulations. The appropriate renormalization procedure required in order to self-consistently accommodate external inputs stemming from other functional approaches or the lattice is discussed in detail, and the value of the gauge coupling is accurately determined at two vastly separated renormalization group scales. Our analysis establishes a clear hierarchy among the vertex form factors. We identify only three dominant ones, in agreement with previous results. The components of the quark propagator obtained from our approach are in excellent agreement with the results from SchwingerDyson equations, the functional renormalization group, and lattice QCD simulation, a simple benchmark observable being the chiral condensate in the chiral limit, which is computed as $(245 \mathrm{MeV})^{3}$. The present approach has a wide range of applications, including the self-consistent computation of bound-state properties and finite temperature and density physics, which are briefly discussed.
\end{abstract}

DOI: 10.1103/PhysRevD.103.094013

\section{INTRODUCTION}

In functional approaches to QCD, the task of computing quark-, gluon-, and hadron-correlation functions is formulated in terms of closed coupled diagrammatic relations between them, which must then be solved numerically. In all these approaches, such as Schwinger-Dyson equations (SDEs), functional renormalization group (fRG), $n$-particle irreducible methods (nPI), and bound state methods [BetheSalpeter (BS), Faddeev- and higher-order equations], the diagrammatic relations are built out of the propagators of the fundamental and composite QCD fields. For reviews on functional methods in QCD, see, e.g., [1-7] (SDEs), [8-13] (fRG), and [14-16] (bound states).

Functional approaches allow for an attractively simple and versatile access to the dynamical mechanisms that drive numerous fundamental QCD phenomena. Moreover, their flexibility in using as external inputs correlation functions

Published by the American Physical Society under the terms of the Creative Commons Attribution 4.0 International license. Further distribution of this work must maintain attribution to the author(s) and the published article's title, journal citation, and DOI. Funded by SCOAP . stemming from distinct nonperturbative setups (e.g., lattice [17-32]) is a particularly welcome feature, which increases their quantitative reliability and their range of applicability. However, such inputs are not always available, prominent and important examples being QCD at finite temperature and density, as well as the hadron spectrum. Hence, in the past two decades, functional methods have evolved into a self-contained quantitative approach to QCD, allowing for quantitative predictions within a "first principle" framework, without the need of external inputs.

This ongoing progress requires quantitative computations involving the full tensor structure of correlation functions, and in particular that of the three- and fourpoint functions, that dominantly drive the dynamics of QCD. Specifically, the quark-gluon vertex is the pivotal ingredient of the matter dynamics of QCD, being intimately connected with fundamental phenomena such as chiral symmetry breaking and quark mass generation, bound state formation, e.g., [33-42], and the QCD phase structure at finite temperature and chemical potential, e.g., [43-62].

To date, the quark-gluon vertices employed in most SDE studies are not based on a full solution of the corresponding dynamical equations, but are rather put together from quark and ghost dressing functions with the aid of the 
Slavnov-Taylor identities (STIs) (see, e.g., [28,63-70]) or rely on perturbative expansion schemes (see, e.g., [71-73]). These are operationally simple and suggestive treatments, with an impressive array of very successful applications, ranging from the properties of hadrons to the phase structure of QCD. However, within the STI constructions, the strength associated with the classical tensor structure requires a phenomenological infrared enhancement, whose size is adjusted by means of the constituent quark masses. The latter, including their momentum dependence, are equivalent to the physical amount of chiral symmetry breaking, and hence, in such an approach, the quantitative strength of chiral symmetry breaking is a phenomenological input rather than a prediction. To be sure, the need for such an enhancement may be attributed to the insufficient knowledge of some of the ingredients comprising these STIs (e.g., quark-ghost kernel [68]). Nonetheless, in view of the results in the present work, as well as of previous considerations within functional approaches [34,60,61, 74-82], it seems to originate mainly from the omission of important tensor structures that are simply not accessible through the standard STI construction.

This situation calls for a self-consistent treatment of the full quark-gluon vertex within the SDE formalism in the Landau gauge. The determination of the eight relevant form factors from their dynamical equations requires the solution of the coupled system of gluon, ghost, and quark propagators, the quark-gluon vertex, as well as additional vertices. The most complete results in this direction have been obtained within functional methods for two-flavor QCD; see [76,77] (quenched), and [79,81] (unquenched). Recently, the fRG results of [81] have been used as input for a $2+1$-flavor analysis within the SDE approach, both in the vacuum and at finite temperature and density $[60,61]$. Despite all these advances, we still lack a well-defined calculational SDE scheme, where one could unambiguously identify and reliably compute the dominant components of this vertex, either self-consistently or with the aid of a given input.

In the present work we put forward a systematic approximation scheme for the set of functional equations governing the quark sector of QCD, by studying in detail the coupled system of SDEs for the quark propagator (quark gap equation) and the quark-gluon vertex. Our SDE analysis reveals that the quark dynamics is dominated by three specific tensor structures of the quark-gluon vertex, in agreement with earlier considerations $[60,61,76,77,79,81]$. It is important to stress that, apart from the dressing associated with the classical tensor, the other two dominant dressings are not accessible by means of an STI-based construction. In fact, the numerical impact of these latter dressings at the level of the gap equations is crucial, furnishing directly the required amount of chiral symmetry breaking without the need to resort to artificial enhancing factors. In our opinion, this demonstrates conclusively that no artificial enhancement is required once the contributions from the appropriate tensorial structures have been properly taken into account. Importantly, we also find that certain tensor structures, which in previous STI treatments seemed dominant precisely due to the use of such enhancing factors, turn out to be clearly subleading. Consequently, the present detailed analysis enables us to restrict our considerations to the three most relevant tensors, thus arriving at a reduced set of fully coupled SDEs, which are solved iteratively together with the quark gap equation.

A central ingredient of the system of equations considered in this work is the gluon propagator, entering in both the gap equation and the SDE for the quark-gluon vertex. The gluon propagator obeys its own SDE [1-5,7], which depends on the quark propagator and further correlation functions, a fact that leads to a proliferation of coupled equations. Even though the complete treatment of such an extended system has already been implemented for $N_{f}=2$ flavor QCD [77,81], in the present work we prefer to maintain the focus on the novel features of our approach rather than be sidetracked by a technically exhaustive analysis. To that end, we treat the gluon propagator as an external ingredient: within our most elaborate and trustworthy approximation, we consider a renormalization point at large, perturbative, momenta with $\mu=40 \mathrm{GeV}$, and use the SDE data for the gluon propagator from $[60,61]$ as external input. These SDE data are based on the fRG twoflavor computation of [55], as are the gluon data of [58], which are also used as input, for the purpose of estimating our systematic error. Finally, we also consider gluon data from $N_{f}=2+1$ lattice simulations $[31,83,84]$ and a renormalization point of $\mu=4.3 \mathrm{GeV}$ for comparison. While the lattice data offer the smallest systematic error, their momentum range is only $p \lesssim 5 \mathrm{GeV}$. As we will see, all the different inputs lead to quantitatively compatible results.

Note also that the gluon propagator is rather insensitive to the details of the quark dynamics, within the range of pion and current quark masses considered here; for a detailed evaluation in the two-flavor case and pion masses in the range $m_{\pi} \approx 0-300 \mathrm{MeV}$ [81]. To be sure, this property does not persist when additional families of active quarks are added to the theory, since, in this case, one implements effectively a transition from infinite to finite quark masses. In fact, as has been clearly established in the analysis of [85], the sequential inclusion of quark families affects the quantitative behavior of the gluon propagator, markedly suppressing its infrared support.

In addition, the results obtained are particularly stable under vast changes in the value of the renormalization point $\mu$. Finally, a chief advantage of this scheme is its relative operational simplicity and low computational cost, combined with quantitative reliability and systematic error control.

The article is organized as follows. In Sec. II we review some general features of the SDE and fRG approaches, and 
introduce the notation that will be used in this work. In Sec. III we set up the gap equation and discuss its renormalization. Then, in Sec. IV we focus on the quarkgluon vertex, present the tensorial basis that will be employed, and derive the system of integral equations satisfied by its form factors. In Sec. V we present a detailed discussion of how to implement self-consistently the renormalization of the SDEs when an external input is employed. In Sec. VI we discuss the procedure that fixes the values of the current quark masses and introduce the light chiral condensate as our benchmark observable. In Sec. VII we present and discuss the central results of our analysis, with special emphasis on the quark mass and the eight form factors of the quark-gluon vertex, evaluated at the symmetric point. Then, in Sec. VIII we confirm the stability of our results under variations of the ultraviolet (UV) cutoff, the renormalization point, and the inputs used for the gluon propagator. In Sec. IX we capitalize on the hierarchy displayed among the vertex form factors and propose a simplified treatment that reduces the numerical cost without compromising the accuracy of the results. In Sec. $X$ we summarize our approach and present our conclusions. Finally, we relegate in two appendixes the discussion of various technical points.

\section{GENERAL CONSIDERATIONS}

In this section we briefly comment on certain important aspects of functional approaches that are relevant for the ensuing analysis, and we introduce the notation that will be employed in this work.

\section{A. The action}

The starting point is the classical action of QCD in covariant gauges, given by

$$
\begin{aligned}
S[\phi]= & \int d^{4} x\left[\frac{1}{4}\left(F_{\mu \nu}^{a}\right)^{2}+\bar{q}\left(\not D+m_{q}\right) q\right. \\
& \left.+\frac{1}{2 \xi}\left(\partial_{\mu} A_{\mu}^{a}\right)^{2}-\bar{c}^{a} \partial_{\mu} D_{\mu}^{a b} c^{b}\right],
\end{aligned}
$$

where the ghost has a positive dispersion, typically used in fRG applications to QCD; for a recent review see [13]. The covariant derivative, $D_{\mu}$, and the field strength tensor, $F_{\mu \nu}$, are given by

$$
\begin{aligned}
F_{\mu \nu}^{a} & =\partial_{\mu} A_{\nu}^{a}-\partial_{\nu} A_{\mu}^{a}+g_{s} f^{a b c} A_{\mu}^{b} A_{\nu}^{c}, \quad \text { and } \\
D_{\mu} & =\partial_{\mu}-i g_{s} A_{\mu}^{a} t^{a}, \quad\left[t^{a}, t^{b}\right]=i f^{a b c} t^{c} .
\end{aligned}
$$

The first two terms in (2.1) are the Yang-Mills and Dirac actions, respectively; in the latter we have suppressed the summation over group indices in the fundamental representation, as well as Dirac and flavor indices. The remaining terms in (2.1) encode the gauge fixing and ghost sector.
In (2.2), the covariant derivative in the fundamental representation reads $\partial_{\mu}-i g_{s} A_{\mu}^{a} T^{a}$, where $T^{a}$ are the corresponding generators, while that of the adjoint representation is given by $\partial_{\mu} \delta^{a b}-g_{s} f^{a b c} A_{\mu}^{c}$. The computations in the present work are carried out in the Landau gauge, $\xi=0$.

\section{B. SDE setup and renormalization}

In contradistinction to the flow equations of the fRG approach, the SDEs depend also on derivatives of the classical QCD action in (2.1). More specifically, we need the bare action, whose parameters absorb the UV infinities of the diagrams. The mapping from bare fields, $\phi^{(0)}$, to renormalized finite fields, $\phi$, is given by

$A_{\mu}^{(0)}=Z_{3}^{1 / 2} A_{\mu}, \quad c^{(0)}=\tilde{Z}_{3}^{1 / 2} c, \quad \bar{c}^{(0)}=\tilde{Z}_{3}^{1 / 2} \bar{c}$,

$q^{(0)}=Z_{2}^{1 / 2} q, \quad \bar{q}^{(0)}=Z_{2}^{1 / 2} \bar{q}$,

while for the strong coupling, masses, and gauge fixing parameters we have, correspondingly,

$g_{s}^{(0)}=Z_{g} g, \quad m_{q}^{(0)}=Z_{m_{q}} m_{q}, \quad \xi^{(0)}=Z_{\xi} \xi$.

Then, the bare QCD action, $S_{\text {bare }}$, reads in terms of the renormalized fields and coupling parameters,

$$
\begin{aligned}
S_{\text {bare }}\left[\phi^{(0)} ; g_{s}^{(0)}, m_{q}^{(0)}\right]= & S\left[Z_{3}^{1 / 2} A_{\mu}, \tilde{Z}_{3}^{1 / 2} c, \tilde{Z}_{3}^{1 / 2} \bar{c}, Z_{2}^{1 / 2} q,\right. \\
& \left.Z_{2}^{1 / 2} \bar{q}, Z_{g} g_{s}, Z_{m_{q}} m_{q}\right] .
\end{aligned}
$$

From (2.3c) we may define the renormalization constants of the three-gluon vertex, $Z_{1}$, the four-gluon vertex, $Z_{4}$, the ghost-gluon vertex, $\tilde{Z}_{1}$, and the quark-gluon vertex, $Z_{1}^{f}$, and we elate them as

$$
\begin{aligned}
& Z_{1}=Z_{g} Z_{3}^{3 / 2}, \quad Z_{4}=Z_{g}^{2} Z_{3}^{2}, \quad \tilde{Z}_{1}=Z_{g} Z_{3}^{1 / 2} \tilde{Z}_{3}^{1 / 2}, \\
& Z_{1}^{f}=Z_{g} Z_{3}^{1 / 2} Z_{2} .
\end{aligned}
$$

\section{C. fRG setup}

The central object of functional approaches to QCD is the one-particle irreducible (1PI) effective action, $\Gamma[\phi]$, where $\phi$ is a superfield, whose components are the fundamental renormalized fields of QCD, including the auxiliary ghost field introduced through the gauge fixing,

$$
\phi=\left(A_{\mu}, c, \bar{c}, q, \bar{q}\right) .
$$

While this is typically rather implicit in most SDE applications, it is commonly the starting point in fRG studies. Derivatives of the effective action $\Gamma[\phi]$ with respect to the fields are the 1PI $n$-point correlation functions, denoted by 


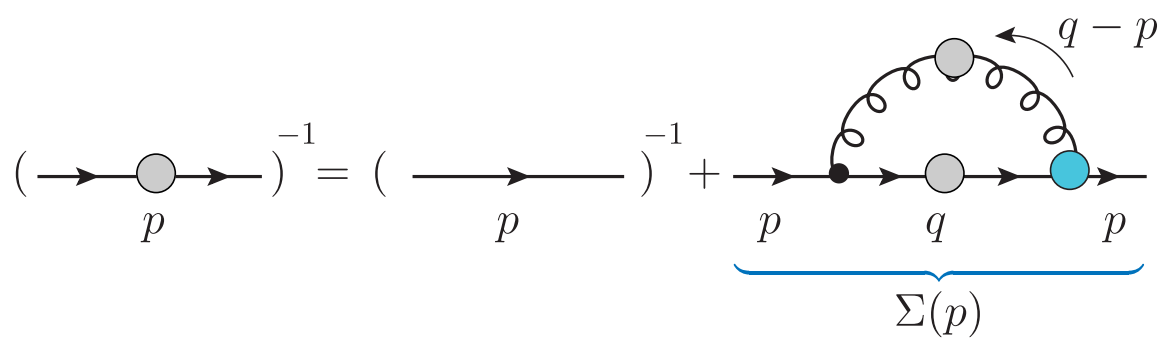

FIG. 1. Diagrammatic representation of the quark gap equation. Gray (blue) circles denote full propagators (vertices), and black dots denote classical vertices.

$$
\Gamma_{\phi_{1} \cdots \phi_{n}}^{(n)}\left(p_{1}, \ldots, p_{n}\right)=\frac{\delta^{n} \Gamma}{\delta \phi_{1}\left(p_{1}\right) \cdots \phi_{n}\left(p_{n}\right)},
$$

where all momenta are considered as incoming. Vertices $\Gamma^{(n)}$ are expanded in a complete tensor basis $\left\{\mathcal{T}_{\phi_{i_{1}} \cdots \phi_{i_{n}}}^{(i)}\right\}$, the standard fRG notation in QCD being

$$
\begin{aligned}
& \Gamma_{\phi_{i_{1}} \cdots \phi_{i_{n}}}^{(n)}\left(p_{1}, \ldots, p_{n}\right) \\
& \quad=\sum_{i} \lambda_{\phi_{i_{1}} \cdots \phi_{i_{n}}}\left(p_{1}, \ldots, p_{n}\right) \mathcal{T}_{\phi_{i_{1}} \cdots \phi_{i_{n}}}^{(i)}\left(p_{1}, \ldots, p_{n}\right),
\end{aligned}
$$

with $\lambda_{\phi_{i_{1}} \cdots \phi_{i_{n}}}$ denoting the scalar form factors (dressings).

Note that the renormalization factors defined in (2.3) have a natural relation to the full dressings of the primitively divergent $n$-point functions in the fRG approach, $Z_{\phi_{i}, k}(p), M_{q, k}(p)$, defined in (2.7), and $\lambda_{\phi_{i_{1}} \cdots \phi_{i_{n}}, k}^{(1)}$, defined in $(2.5 \mathrm{~b})$; for a detailed account see $[8,13]$.

\section{Running couplings}

We next consider the different "avatars" of the strong running coupling $\alpha_{s}(\bar{p})=g_{s}^{2}(\bar{p}) / 4 \pi$, which can be deduced from the form factors $\lambda^{(1)}$ associated with the classical tensor structures of the four fundamental QCD vertices. In particular, in the present analysis we will employ the running couplings obtained from the ghostgluon and quark-gluon vertices, given by

$\alpha_{c \bar{c} A}(\bar{p})=\frac{1}{4 \pi} \frac{\left[\lambda_{c \bar{c} A}^{(1)}(\bar{p})\right]^{2}}{Z_{A}(\bar{p}) Z_{c}^{2}(\bar{p})}, \quad \alpha_{q \bar{q} A}(\bar{p})=\frac{1}{4 \pi} \frac{\left[\lambda_{q \bar{q} A}^{(1)}(\bar{p})\right]^{2}}{Z_{A}(\bar{p}) Z_{q}^{2}(\bar{p})}$,

where $\bar{p}$ is a symmetric-point configuration and $Z_{A}, Z_{c}$, and $Z_{q}$ are the dressings of the two-point functions (suppressing color),

$$
\begin{aligned}
\Gamma_{A A \mu \nu}^{(2)}(p) & =Z_{A}(p) p^{2} P_{\mu \nu}(p)+\frac{1}{\xi} p_{\mu} p_{\nu}, \\
\Gamma_{c \bar{c}}^{(2)}(p) & =Z_{c}(p) p^{2}, \\
\Gamma_{q \bar{q}}^{(2)}(p) & =Z_{q}(p)\left[i \not p+M_{q}(p)\right],
\end{aligned}
$$

where we have introduced the transverse projection operator

$$
P_{\mu \nu}(p)=\delta_{\mu \nu}-\frac{p_{\mu} p_{\nu}}{p^{2}}
$$

usually denoted by $\Pi_{\mu \nu}^{\perp}(p)$ in the fRG literature. Note that the above two-point functions are the inverses of the gluon, ghost, and quark propagators, respectively.

By virtue of the fundamental STIs of the theory, all QCD couplings coincide for large values of $\bar{p}$,

$$
\begin{aligned}
\alpha_{i}(\bar{p}) & =\alpha_{s}(\bar{p}), \\
i & =\left(c \bar{c} A, q \bar{q} A, A^{3}, A^{4}\right) \text { for perturbative } \bar{p}=\bar{p}_{\text {pert }} .
\end{aligned}
$$

As the momentum $\bar{p}$ gets smaller, the various $\alpha_{i}(\bar{p})$ start deviating from each other, due to differences induced by nontrivial contributions from the scattering kernels appearing in the STIs. As has been pointed out in [81], the amount of chiral symmetry breaking obtained from the gap equation appears to be particularly sensitive to the UV coincidence of the couplings described by (2.9). The preservation of (2.9) is an indispensable feature of any quantitatively reliable framework; in particular, special truncation schemes such as the pinch technique background field method [5] are tailor-made for this task.

\section{THE QUARK GAP EQUATION}

The quark gap equation [1-7] relates the inverse quark propagator, $\Gamma_{q \bar{q}}^{(2)}(p)$, to its classical counterpart, $S_{q \bar{q}}^{(2)}(p)$, to the quark and gluon propagators, and the classical and full quark-gluon vertices; see Fig. 1. Schematically it reads

$$
\begin{aligned}
& \Gamma_{q \bar{q}}^{(2)}(p) \\
& \quad=S_{q \bar{q}}^{(2)}+Z_{1}^{f} g_{s} \int_{q} G_{A A}(q-p)(-i \gamma) G_{q \bar{q}}(q) \Gamma_{q \bar{q} A}^{(3)}(q,-p),
\end{aligned}
$$

where we suppress all Lorentz and color indices, and $g_{s}$ stands for the gauge coupling. The four-dimensional momentum integration has been abbreviated by 


$$
\int_{q}:=\int_{\operatorname{reg}} \frac{d^{4} q}{(2 \pi)^{4}}
$$

where the subscript "reg" indicates a suitable regularization of the momentum integral; common choices include the dimensional regularization or an appropriately implemented momentum cutoff. The respective cutoff parameter (e.g., $\epsilon$ or $\Lambda^{2}$ ) appears also in all renormalization constants, and in particular the quark-gluon vertex renormalization, $Z_{1}^{f}$, as well as the wave function renormalization, $Z_{2}$, and the mass renormalization, $Z_{m_{q}}$, of the quark. The last two factors enter into (3.1) through $S_{q \bar{q}}^{(2)}(p)$, the second derivative of the bare QCD action, (2.3c), with respect to the renormalized quark and antiquark fields [see (2.3a)],

$$
S_{q \bar{q}}^{(2)}(p)=i Z_{2} \not p+Z_{m_{q}} m_{q},
$$

where $m_{q}$ denotes the bare current quark mass.

The full gluon propagator, $G_{A A \mu \nu}^{a b}(p)$, in the Landau gauge, and the quark propagator, $G_{q \bar{q}}^{a b}(p)$, are given by

$$
G_{A A \mu \nu}^{a b}(p)=\delta^{a b} P^{\mu \nu}(p) G_{A}(p), \quad G_{q \bar{q}}^{a b}(p)=\delta^{a b} G_{q}(p) .
$$

In (3.4), $G_{A}(p)$ is the scalar part of the gluon propagator, and $G_{q}(p)$ carries only the Dirac structure but not the trivial color structure. Both $G_{A}(p)$ and $G_{q}(p)$ can be described in terms of the scalar dressings introduced in (2.7), to wit,

$G_{A}(p)=\frac{1}{Z_{A}(p) p^{2}}, \quad G_{q}(p)=\frac{1}{Z_{q}(p)\left[i \not p+M_{q}(p)\right]}$,

where $M_{q}(p)$ is the momentum-dependent mass function. Note that in the fRG approach, for large cutoff scales, the functions $Z_{A}(p)$ and $Z_{q}(p)$ tend toward the corresponding (finite) wave function renormalizations, while $M_{q}(p)$ tends to the bare quark mass.

Finally, $\left[\Gamma_{\bar{q} q A}^{(3)}\right]_{\nu}^{a}(q,-p)$ denotes the quark-gluon vertex, in accordance with the general definition of (2.5), with all momenta considered as incoming.

The presence of the transverse projection operator $P_{\mu \nu}$ in (3.1) makes natural the use of the transversely projected version of the quark-gluon vertex. Specifically, for the purposes of the present work we introduce the transversely projected vertex $\boldsymbol{\Gamma}_{\mu}(q,-p)$, defined through

$$
P_{\mu \nu}(p-q)\left[\Gamma_{\bar{q} q A}^{(3)}\right]_{\nu}^{a}(q,-p)=\mathbb{1}_{\mathrm{f}} T_{\mathrm{c}}^{a} \boldsymbol{\Gamma}_{\mu}(q,-p),
$$

where $\mathbb{1}_{\mathrm{f}}$ denotes the identity matrix in flavor space. Note that while $\left[\Gamma_{\bar{q} q A}^{(3)}\right]_{\nu}^{a}(q,-p)$ requires 12 tensors for its full decomposition, $\boldsymbol{\Gamma}_{\mu}(q,-p)$ is comprised by a subset of only eight; for more details, see, e.g., [77,81].
With the above definitions, the color contractions in (3.1) can easily be carried out, and we arrive at the standard form of the gap equation,

$$
Z_{q}(p)\left[i \not p+M_{q}(p)\right]=Z_{2} i \not p+Z_{m_{q}} m_{q}+\Sigma(p),
$$

with the renormalized self-energy

$$
\begin{aligned}
\Sigma(p)= & Z_{1}^{f} g_{s} C_{f} \int_{q} \frac{1}{Z_{A}(q-p)(q-p)^{2}} \gamma_{\mu} \\
& \times \frac{1}{Z_{q}(q)\left[i \not q+M_{q}(q)\right]} \boldsymbol{\Gamma}_{\mu}(q,-p),
\end{aligned}
$$

where $C_{f}$ denotes the Casimir eigenvalue of the fundamental representation, with $C_{f}=4 / 3$ for $S U(3)$.

Note that Eq. (3.7) is finite due to the regularization of the loop integral, as indicated in (3.2). As mentioned there, the cutoff dependences of the loop integral and of $Z_{2}, Z_{m_{q}}$, and $Z_{1}^{f}$ cancel against each other, giving finally rise to cutoff-independent functions $Z_{q}(p)$ and $M_{q}(p)$. As we discuss in the next section, an analogous renormalization procedure renders the vertex $\boldsymbol{\Gamma}_{\mu}(q,-p)$ cutoff independent.

The gap equation in (3.7) can be projected on its Dirac vector and scalar parts by multiplying it with either $\mathbb{1}$ or $\not p$ and performing the corresponding traces. This leads us to the standard set of coupled SDEs for $Z_{q}(p)$ and $M_{q}(p)$,

$$
\begin{aligned}
Z_{q}(p) p^{2} & =Z_{2} p^{2}-Z_{1}^{f} \operatorname{tr}[i \not p \Sigma(p)], \\
M_{q}(p) & =Z_{q}^{-1}(p)\left(Z_{m_{q}} m_{q}+Z_{1}^{f} \operatorname{tr}[\Sigma(p)]\right) .
\end{aligned}
$$

We next specify the renormalization conditions at a given renormalization scale $\mu$. As is common to functional approaches, we employ a nonperturbative version of the momentum subtraction (MOM) scheme, where the renormalized quantum corrections of all primitively divergent vertices with momenta $p_{1}, \ldots, p_{n}$ vanish at a symmetric point $\bar{p}^{2}=\mu^{2}$, when

$$
p_{i}^{2}=\bar{p}^{2}, \quad \forall i=1, \ldots, n .
$$

In particular, the dressings of the two-point functions reduce to unity,

$$
Z_{A}(\mu)=1, \quad Z_{c}(\mu)=1, \quad Z_{q}(\mu)=1, \quad M_{q}(\mu)=m_{q},
$$

where $Z_{c}(p)$ is the dressing associated with the ghost propagator. Similarly, in the case of the vertices, the symmetric point dressings $\left.\lambda_{\phi_{1} \cdots \phi_{n}}^{(1)}(\bar{p}):=\lambda_{\phi_{1} \cdots \phi_{n}}^{(1)}\left(p_{1}, \ldots, p_{n}\right)\right)\left.\right|_{p_{i}^{2}=\bar{p}^{2}}$ of the classical tensor structures satisfy 

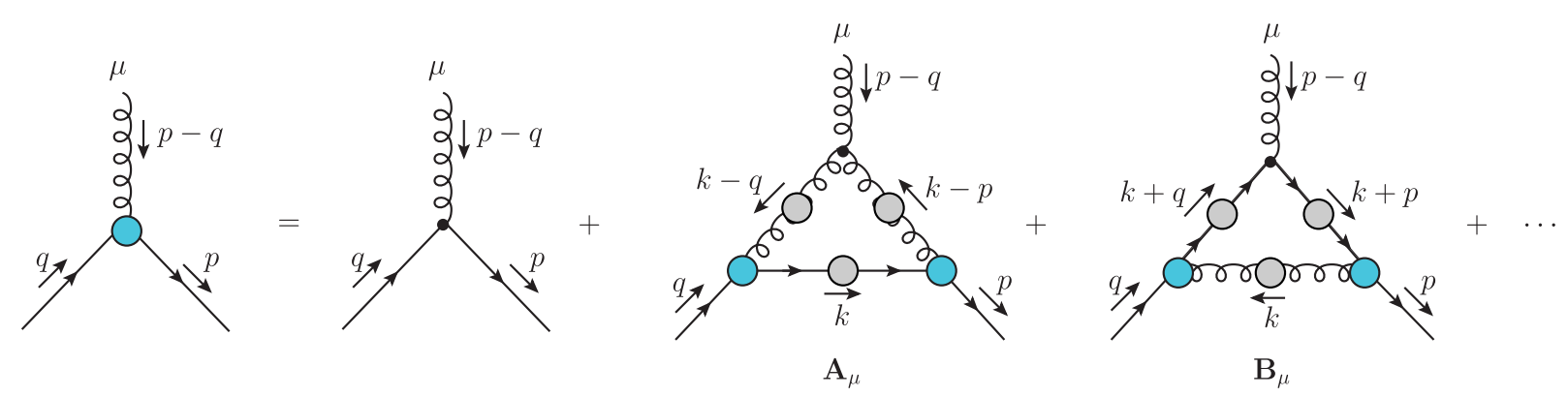

FIG. 2. Diagrammatic representation of the quark-gluon SDE. Gray (blue) circles denote full propagators (vertices), and black dots denote classical vertices. The ellipses denote higher-order contributions: diagrams without perturbative one-loop counterparts.

$$
\begin{array}{ll}
\lambda_{A^{3}}^{(1)}(\mu)=g_{s}, & \lambda_{A^{4}}^{(1)}(\mu)=g_{s}^{2}, \\
\lambda_{c \bar{c} A}^{(1)}(\mu)=g_{s}, & \lambda_{q \bar{q} A}^{(1)}(\mu)=g_{s} .
\end{array}
$$

Evidently, all renormalization constants also depend on the subtraction point $\mu$.

Within the renormalization scheme defined above, we have that $\Gamma_{q \bar{q}}\left(p^{2}=\mu^{2}\right)=i \not p+m_{q}$, and in the standard MOM scheme the respective renormalization factors would be given by

$Z_{2}=1+\left.\frac{\operatorname{tr}\left[i \not p Z_{1}^{f} \Sigma(p)\right]}{p^{2}}\right|_{p^{2}=\mu^{2}}, \quad Z_{m_{q}}=1-\left.\frac{\operatorname{tr}\left[Z_{1}^{f} \Sigma(p)\right]}{m_{q}}\right|_{p^{2}=\mu^{2}}$.

In the present work we resort to a MOM-type scheme, by using (3.11b) and a minor modification of (3.12), implemented by a rescaling of the field and triggered by the fRG input data. This is discussed further in Sec. V and in Sec. A and in particular in Sec. A 2.

The solution of the quark gap equation requires the knowledge of the gluon propagator and the quark-gluon vertex, which, in turn, depend on the quark propagator and further correlation functions, thus leading to an extended system of coupled integral equations, which must be solved simultaneously. Such a complete, fully back-coupled analysis, subject to certain simplifying approximations, is indeed feasible and has been presented within functional approaches for $N_{f}=2$ flavor QCD in [77,81]. However, the main purpose of the present work is the detailed analysis of the system of quark propagator and quarkgluon vertex, as well as the discussion of quantitative approximation schemes. For this reason we opt for a simpler treatment, which permits us to maintain our focus on the novel aspects of our approach. In particular, the gluon propagator entering into both the gap equation and the vertex SDE will be treated as an external ingredient. Thus, rather than solving its own dynamical equation, we will employ the results obtained in the unquenched lattice simulations of $[31,83,84]$ and the functional analysis of $[55,58]$.

\section{SDE OF THE QUARK-GLUON VERTEX}

In this section we set up and discuss the SDE for the $\boldsymbol{\Gamma}_{\mu}$ defined in (3.6), which enters in the quark gap equation. In the present work we consider the "one-loop dressed" approximation of this SDE, which is diagrammatically depicted in Fig. 2. The terms omitted from this SDE correspond to terms that do not lead to perturbative oneloop contributions. All such graphs may be systematically accounted for by carrying out the so-called "skeleton expansion" of the relevant kernels. In particular, the two graphs depicted in Fig. 2 correspond to the lowest order terms in the skeleton expansion of the kernels $\bar{q} q A A$ and $\bar{q} q \bar{q} q$. This functional equation will be projected on its different tensorial components, thus furnishing a set of dynamical equations governing the respective form factors.

The SDE for the vertex $\boldsymbol{\Gamma}_{\mu}$ is expressed as

$$
\begin{aligned}
\boldsymbol{\Gamma}_{\mu}(q,-p)= & Z_{1}^{f} g_{s} P_{\mu \nu}(p-q)\left(-i \gamma_{\nu}\right) \\
& +\mathbf{A}_{\mu}(q,-p)+\mathbf{B}_{\mu}(q,-p),
\end{aligned}
$$

with the contributions of the graphs $\mathbf{A}_{\mu}(q,-p)$ and $\mathbf{B}_{\mu}(q,-p)$ in Fig. 2 given by

$$
\begin{aligned}
& \mathbf{A}_{\mu}(q,-p)=\frac{Z_{1} N_{c}}{2} P_{\mu \nu}(p-q) \int_{k} \Gamma_{\nu \alpha \beta}^{(0)} G_{A}(k-q) G_{A}(k-p) \boldsymbol{\Gamma}_{\alpha}(k,-p) G_{q}(k) \boldsymbol{\Gamma}_{\beta}(q,-k), \\
& \mathbf{B}_{\mu}(q,-p)=-\frac{Z_{1}^{f}}{2 N_{c}} P_{\mu \nu}(p-q) \int_{k} G_{A}(k) \boldsymbol{\Gamma}_{\alpha}(k+p,-p) G_{q}(k+p)\left(-i \gamma_{\nu}\right) G_{q}(k+q) \boldsymbol{\Gamma}_{\alpha}(q,-k-q) .
\end{aligned}
$$


In the above formulas, $N_{c}=3$ for $S U(3)$, the vertex renormalization constants $Z_{1}$ and $Z_{1}^{f}$ were defined after (2.3c), and $\Gamma_{\nu \alpha \beta}^{(0)}$ denotes the classical three-gluon vertex,

$$
\begin{aligned}
\Gamma_{\nu \alpha \beta}^{(0)}= & g_{s}\left[(2 k-p-q)_{\nu} g_{\alpha \beta}+(2 q-p-k)_{\alpha} g_{\nu \beta}\right. \\
& \left.+(2 p-q-k)_{\beta} g_{\alpha \nu}\right],
\end{aligned}
$$

where we have factored out the color factor $f^{a b c}$.

The vertex $\boldsymbol{\Gamma}_{\mu}$ may be decomposed in a basis formed by the transverse projections $P_{\mu \nu} \mathcal{T}_{i}^{\mu}$ of eight independent tensorial structures, denoted by $\mathcal{T}_{i}^{\mu}$, which can be derived from gauge-invariant quark-gluon operators [77,81], according to

$$
\begin{aligned}
\bar{q} \not D q & \rightarrow \mathcal{T}_{1}^{\mu}, \quad \bar{q} \not D^{2} q \rightarrow \mathcal{T}_{2}^{\mu}, \mathcal{T}_{3}^{\mu}, \mathcal{T}_{4}^{\mu}, \\
\bar{q} \not D^{3} q & \rightarrow \mathcal{T}_{5}^{\mu}, \mathcal{T}_{6}^{\mu}, \mathcal{T}_{7}^{\mu}, \quad \bar{q} \not D^{4} q \rightarrow \mathcal{T}_{8}^{\mu} .
\end{aligned}
$$

The full tensor basis with 12 elements is then given in terms of transverse and longitudinal projections of the tensors (4.4). Specifically, introducing $P_{\mu \nu}^{\mathrm{L}}:=\delta_{\mu \nu}-P_{\mu \nu}$, a concrete choice is given by $[77,81]$

$$
\left(\left\{P_{\mu \nu} \mathcal{T}_{i}^{\mu}\right\}, P_{\mu \nu}^{\mathrm{L}} \mathcal{T}_{1,2,6,8}^{\mu}\right),
$$

where the projection operators $P_{\mu \nu}$ and $P_{\mu \nu}^{\mathrm{L}}$ carry the gluon momentum.

In particular, for the transversally projected quark gluon vertex we have

$\boldsymbol{\Gamma}^{\mu}(q,-p)=\sum_{i=1}^{8} \lambda_{i}(q,-p) P^{\mu \nu}(q-p) \mathcal{T}_{i}^{\nu}(q,-p)$,

where the shorthand notation $\lambda_{i}:=\lambda_{q \bar{q} A}^{(i)}$ was introduced.

With the aid of (4.6), and through appropriate tensor contractions, the starting SDE of (4.1) may be converted into a system of coupled integral equations for $\lambda_{i}(p, q)$. Specifically, one obtains

$$
\lambda_{i}(q,-p)=Z_{1}^{f} g_{s} \delta_{i 1}+a_{i}(q,-p)+b_{i}(q,-p), \quad i=1, \ldots, 8
$$

with

$$
\begin{aligned}
a_{i}(q,-p)= & \frac{Z_{1} N_{c}}{2} \int \frac{d^{4} k}{(2 \pi)^{4}} \lambda_{j}(k,-p) \lambda_{k}(q,-k) G_{A}(k-q) \\
& \times G_{A}(k-p) K_{i j k}(p, q, k), \\
b_{i}(q,-p)= & -\frac{Z_{1}^{f}}{2 N_{c}} \int \frac{d^{4} k}{(2 \pi)^{4}} \lambda_{j}(k+p,-p) \\
& \times \lambda_{k}(q,-k-q) G_{A}(k) \tilde{K}_{i j k}(p, q, k),
\end{aligned}
$$

where the kernels $K_{i j k}(p, q, k)$ and $\tilde{K}_{i j k}(p, q, k)$ contain combinations of $Z_{q}, M_{q}$, and the various momenta; further information on their precise structure is provided in Appendix B.

The renormalization condition corresponding to (3.11) dictates that, at the symmetric point $\bar{p}^{2}=\mu^{2}$, we must impose

$$
Z_{1}^{f} g_{s}=g_{s}-\left[a_{1}(q,-p)+b_{1}(q,-p)\right]_{p^{2}=q^{2}=\mu^{2}} .
$$

This leads us to the final, explicitly renormalized coupled integral equations for $\lambda_{i}(p, q)$,

$$
\begin{aligned}
\lambda_{i}(p, q)= & a_{i}(p, q)+b_{i}(p, q) \\
& +\left(g_{s}-\left[a_{i}(q,-p)+b_{i}(q,-p)\right]_{p^{2}=q^{2}=\mu^{2}}\right) \delta_{i 1},
\end{aligned}
$$

which satisfies manifestly (3.11b).

As we will see in detail in Sec. VII, the numerical treatment of the system of coupled integral equations given by Eqs. (3.9), (4.10), and (4.8) reveals a clear hierarchy among the dressings $\lambda_{i}$. In particular, depending on their numerical impact, the $\lambda_{i}$ may be naturally separated into dominant, subleading, and negligible.

Specifically, the three dominant components of the quark gluon vertex are $\lambda_{1,4,7}$, associated with the tensor structures

$\mathcal{T}_{1}^{\mu}(p, q)=-i \gamma^{\mu}, \quad \mathcal{T}_{4}^{\mu}(p, q)=(\not p+\not q) \gamma^{\mu}$,

$\mathcal{T}_{7}^{\mu}(p, q)=\frac{i}{2}[\not p, q] \gamma^{\mu}$.

As we will see in Sec. VII, keeping only these three form factors in the coupled SDE analysis [i.e., the terms corresponding to $i=1,4,7$ in (4.8)] already furnishes quantitatively accurate results for our benchmark observable, the RG-invariant chiral condensate. It is important to emphasize that out of these three dominant structures, only $\lambda_{1}$ is accessible to an STI-based derivation of the quark gluon vertex, in the spirit of the original Ball Chiu (BC) construction.

The three subleading components, $\lambda_{2,5,6}$, are associated with the basis elements

$\mathcal{T}_{2}^{\mu}(p, q)=(q-p)^{\mu}, \quad \mathcal{T}_{5}^{\mu}(p, q)=i(\not p+\not q)(p-q)^{\mu}$,

$\mathcal{T}_{6}^{\mu}(p, q)=i(\not p-\not q)(p-q)^{\mu}$.

These three dressings may be obtained from the STI-based constructions, implemented only in the vacuum. Therefore, in view of the numerous applications to QCD at finite temperature and density, SDE-based computations of these subleading tensor structures, such as the one put forth here, are clearly preferable. 
Finally, the form factors associated with the tensors

$$
\mathcal{T}_{3}^{\mu}(p, q)=(\not p-\not q) \gamma^{\mu}, \quad \mathcal{T}_{8}^{\mu}(p, q)=-\frac{1}{2}[\not p, \not q](p-q)^{\mu}
$$

are negligible, having no appreciable numerical impact on our benchmark observable or any other relevant quantity (see also [60,61]).

This concludes the description of our SDE setup.

\section{EXTERNAL INPUT AND SELF-CONSISTENT RENORMALIZATION}

In this section we discuss self-consistent renormalization schemes for the SDE with a given external input. This issue is addressed both in general and for the given input data for the gluon propagator used here. In addition, we detail the origin and characteristics of these data.

In Sec. VA we elaborate on the implementation of multiplicative renormalization in a MOM-type scheme in the present nonperturbative approach; there, and in Sec. A, we also emphasize the differences to the standard MOM scheme. In Sec. V B we provide an overview on the gluon propagator data used as input, in Sec. V C we discuss the general self-consistent determination of the value of the renormalized coupling $\alpha_{s}(\mu)$ at the renormalization scale $\mu$, and in Sec. V D we determine $\alpha_{s}(\mu)$ for the gluon input data specified in Sec. V C.

\section{A. Multiplicative renormalization}

The self-consistent implementation of multiplicative renormalization at the level of the nonperturbative SDEs constitutes a yet unresolved problem, which has been treated only approximately within numerical applications; see, e.g., [7,36,66,70,86-88]. In the present context, the complications stemming from this issue manifest themselves at the level of the gap equation by the presence of the factor $Z_{1}^{f}$ in the definition of the quark self-energy $\Sigma(p)$, and at the level of the SDE for $\boldsymbol{\Gamma}^{\mu}$ through the factors $Z_{1}$ and $Z_{1}^{f}$ entering in the expressions for $\mathbf{A}_{\mu}$ and $\mathbf{B}_{\mu}$, respectively.

Evidently, the renormalization constants $Z_{1}, Z_{f}$ display a nontrivial ("marginal") dependence on the UV cutoff, which is required for rendering the diagrams finite. However, the order-by-order cancellation known from perturbation theory does not translate straightforwardly to the nonperturbative setup of the SDEs. In this work we adopt a modification of the standard MOM scheme and its approximation used in the SDE. In fact, the present MOMtype scheme is the standard one used in $\mathrm{fRG}$ applications to QCD [58,81], and it has also been used in recent SDE applications $[60,61]$. Our gluon input data are taken from these sources, and hence, the respective MOM-type scheme is the natural one for their implementation. The full setup will be explained elsewhere, but its spirit is entailed in the following consideration: assume that the cutoff dependence encoded in $Z_{1}, Z_{f}$ has been successfully canceled, and set the cutoff-independent finite parts to zero, leading to $Z_{1}, Z_{f} \rightarrow 1$ at the level of the diagrams. Conceptually, this can be achieved by a subtraction of the diagrams at the renormalization point, accompanied by respective rescalings of the fields.

For a large renormalization scale, $\mu \rightarrow \infty$, this procedure can be put forth by invoking asymptotic freedom, $g_{s}(\mu \rightarrow \infty) \rightarrow 0$, and the fact that the finite parts stemming from $Z_{1}, Z_{f} \rightarrow 1$ in the diagrams are proportional to $g_{s}^{2}$. This argument is further supported within the setup with fRG inputs [58,81] and SDE inputs [60,61], which satisfy these RG conditions self-consistently. For more details, including the relations of $\Lambda_{\mathrm{QCD}}$ in the standard MOM scheme and present MOM-type scheme, we refer the reader to Appendix A. As already mentioned above, the use of the fRG input data for the gluon propagator is the main reason for resorting to this modification of the standard MOM scheme, as then the RG condition on the input data and the SDE coincide. Nonetheless, these considerations do not constitute a proof of the full self-consistency of this procedure, which is the subject of ongoing work.

In summary, for the numerical treatment of the system of integral equations presented here, we simply implement the substitution

$$
\begin{aligned}
\Sigma(p) & \left.\rightarrow \Sigma(p)\right|_{Z_{1}^{f}=1}, \\
{\left[a_{i}(p, q), b_{i}(p, q)\right] } & \rightarrow\left[a_{i}(p, q), b_{i}(p, q)\right]_{Z_{1}^{f}=1=Z_{1}} .
\end{aligned}
$$

It is evident from the discussion above that the simplifications implemented by (5.1) are bound to induce a residual cutoff and $\mu$ dependence to the results obtained, which are discussed in Sec. VIII.

\section{B. Gluon propagator}

The gluon propagator can be computed from its own SDE; for the most recent results in Yang-Mills theory, see [88-91], while for 2+1-flavor solutions of the fRGassisted SDE, see [60,61]. Consequently, we could extend the current system to a fully self-coupled one, the only input being the strong coupling and the current quark masses. However, in this work we concentrate rather on the novel key ingredient, namely the computation of the full transversally projected quark-gluon vertex and its properties. Therefore, we simply take quantitative input data from the lattice $[31,83,84]$, the $\operatorname{SDE}[60,61,92,93]$, or the fRG [58].

For the present computation, the input data have to cover the momentum region $p^{2} \in\left[0, \Lambda^{2}\right]$, where $\Lambda$ is the UV cutoff of the loop integrals in the SDEs. In the present work, we consider UV cutoffs in the range $\Lambda=50-5000 \mathrm{GeV}$, as we also test the cutoff independence 

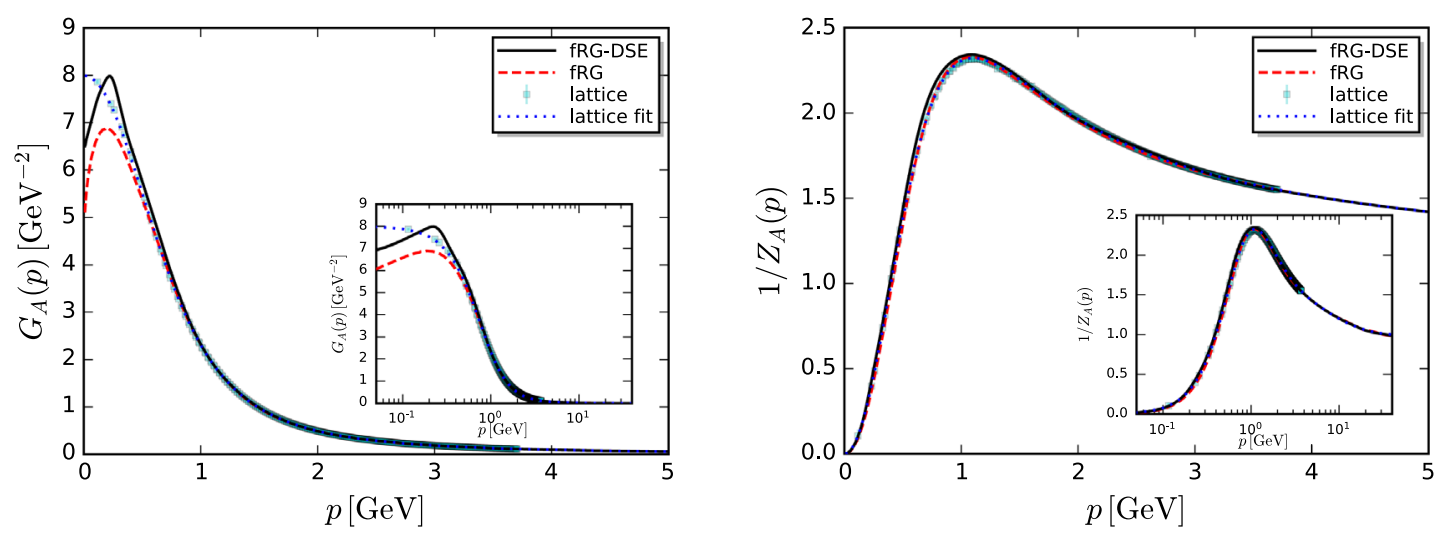

FIG. 3. The $2+1$-flavor gluon propagator, $G_{A}\left(p^{2}\right)$, and dressing function $1 / Z_{A}(p)=p^{2} G_{A}(p)$. Lattice simulations [31,83,84], fRGDSE approach [60,61], and fRG approach [58]. The computations in [58,60,61] are based on the 2-flavor input fRG data from [81].

of the results (see Sec. VIII A). While the functional input data cover the full momentum regime, lattice input data are restricted within $p \lesssim 4 \mathrm{GeV}$. Consequently, they have to be extrapolated toward the UV; the best extrapolation is provided by the functional input data, which agree quantitatively with the lattice data for $p \gtrsim 1 \mathrm{GeV}$.

Next, we provide a physically motivated fit of the resulting "functional-lattice" propagator, valid within the regime $p \in[0,40] \mathrm{GeV}$, which incorporates explicitly the one-loop resummed running of $G_{A}(p)$. In particular,

$$
\begin{aligned}
G_{A}(p)= & \frac{\left(a^{2}+p^{2}\right) /\left(b^{2}+p^{2}\right)}{M^{2}\left(p^{2}\right)+p^{2}\left[1+c \ln \left(d^{2} p^{2}+e^{2} M^{2}\left(p^{2}\right)\right)\right]^{\gamma}}, \\
& \text { with } M^{2}(p)=\frac{f^{4}}{g^{2}+p^{2}}
\end{aligned}
$$

where $\gamma=\left(13-4 / 3 N_{f}\right) /\left(22-4 / 3 N_{f}\right)$ denotes the one-loop anomalous dimension of the gluon propagator in the Landau gauge. The optimized values of the fitting parameters are given by $\{a, b, c, d, e\}=$ $\left\{1 \mathrm{GeV}, 0.735 \mathrm{GeV}, 0.12,0.0257 \mathrm{GeV}^{-1}, 0.081 \mathrm{GeV}^{-1}\right\}$, together with $\{f, g\}=\{0.65 \mathrm{GeV}, 0.87 \mathrm{GeV}\}$. As can be seen in Fig. 3, this fit matches very accurately the input points in the physically relevant region of momenta.

Note also that, as can be seen in Fig. 3, the input data for $G_{A}(p)$ differ in the infrared, i.e., for $p^{2} \lesssim 1 \mathrm{GeV}$ (scaling $[58,60,61]$ vs decoupling or massive $[31,83,84]$; for related discussions, see, e.g., $[92,94,95])$. Nonetheless, the quark propagator obtained using either of them, as well as the computed physical observables, agrees within our systematic error bars. The reason for this is related to the fact that, inside the quantum loops considered here, the gluon propagator $G_{A}(p)$ is eventually multiplied by $p^{2}$; as a result, the infrared differences are largely washed out, and the relevant quantity, $Z_{A}^{-1}(p)=p^{2} G_{A}(p)$, is practically identical for both.

\section{Self-consistent determination of $\alpha_{s}(\boldsymbol{\mu})$}

A necessary ingredient for our analysis is the value of the dressing $\lambda_{1}$ at the symmetric point, which, for sufficiently large values of $\mu$ is equal to the (unique) perturbative $g_{s}$, or the $\alpha_{s}$ defined in (2.6) [see also (3.11b)]. If no external inputs were employed, one would determine $\lambda_{1}$ at $\mu$ by simply imposing that $\alpha_{s}(\mu)$ should coincide with the physical QCD coupling, $\alpha_{s, \text { phys }}$, at a given momentum scale; for example, this can be done at the mass $M_{Z}$ of the $Z$ boson, i.e., $\alpha_{s}(\mu)=\alpha_{s \text {, phys }}\left(M_{Z}\right)$. However, since in our study we use as external input the $N_{f}=2+1$ gluon propagator from lattice and functional methods, the renormalization procedures adopted in those earlier computations need be incorporated into the present SDE treatment, such that a self-consistent value for $\alpha_{s}(\mu)$ may be obtained. This leads us to the MOM-type renormalization scheme used here, as well as within the fRG and SDE computations in $[60,61,77,81,96]$.

The self-consistent calibration of $\alpha_{s}(\mu)$, in any scheme, may be implemented according to two different procedures, (i) and (ii), detailed below. In (i), one compares correlation functions computed within the present SDE setup, whose form depends on the value of $\alpha_{s}$ used, with data for them originating from the same framework that provides the required external input. In (ii), one invokes self-consistency conditions between the results of the current SDE approach and those derived from the STIs.

We emphasize that both procedures are optimized when implemented in the perturbative and semiperturbative regime with

$$
p \gtrsim p_{\text {pert }}, \quad \text { with } \quad p_{\text {pert }} \approx 4 \mathrm{GeV},
$$

where the truncation errors are small and under control; instead, their extension to the nonperturbative infrared regime is bound to worsen the calibration. In fact, while in the regime of (5.3) STI as well as vertex couplings agree at least up to two loops, they deviate markedly from each 
other as $p \rightarrow 0$; see [81]. Moreover, the regularity assumption that is implicit in the direct use of the STIs for the determination of transverse couplings [as in (5.4)] may fail in the infrared; for more details, see [81,96-98].

The concrete implementation of (i) and (ii) is presented in detail below.

(i) The setup in the present work only requires the data for the $2+1$-flavor gluon propagator as external input from either distinct SDE approaches, the fRG or lattice simulations. Within all these frameworks, one has access to datasets not only for this specific input but also for additional correlation functions, as well as derived couplings, e.g., via (2.6). While the latter are not needed as explicit inputs for the SDE, they can be used as a means of calibrating the calculation, because they can be recomputed from their own dynamical equation within the present SDE setup. In doing so, it is clear that their momentum dependence changes as the value of $\alpha_{s}(\mu)$ is varied. Thus, the external datasets may be reproduced for a unique self-consistent choice of $\alpha_{s}(\mu)$, which calibrates our approach (for $p \gtrsim p_{\text {pert }}$ ).

If a propagator is chosen for the purpose of calibration, the ghost propagator is clearly the best choice, as it is governed by a rather simple SDE, whose only other ingredient is the ghost-gluon vertex, which is protected by Taylor's nonrenormalization theorem. For example, if one were to use as external input the gluon propagator from the lattice, the calibration proceeds by computing the ghost dressing function $Z_{c}\left(p^{2}\right)$ within our SDE setup, adjusting the $\alpha_{s}(\mu)$ such that the lattice data for $Z_{c}\left(p^{2}\right)$ will be best reproduced.

If one of the running couplings, $\alpha_{i}(\bar{p})$, is employed for the calibration, we compute its shape both within the approach that furnishes the external input and within the SDE setup. Then, self-consistency requires that the $\operatorname{SDE} \alpha_{s}(\mu)$ is chosen such that the difference between the two $\alpha_{i}(\bar{p})$ 's is minimized, for $\bar{p} \gtrsim p_{\text {pert }}$.

We close with the remark that, in the present setup, all procedures mentioned above lead to $\alpha_{s}(\mu)$ that agree within the small numerical and systematic errors. We consider this an important self-consistency check of the SDE approach put forth here.

(ii) If the additional results needed for the implementation of (i) are unavailable, one can use the STI satisfied by the quark-gluon vertex in order to fix $\alpha_{s}(\mu)$. Specifically, for $\bar{p} \gtrsim p_{\text {pert }}$ one uses the relation

$$
\lambda_{1}(\bar{p})=g_{s}(\mu) L_{1}(\bar{p}),
$$

where $L_{1}$ is the solution of the STI for the longitudinally projected classical tensor structure (see
$[36,81])$. As in (i), minimizing the difference between the two sides of (5.4) singles out a unique $\alpha_{s}(\mu)$.

This concludes our general discussion of the selfconsistent determination of $\alpha_{s}(\mu)$.

\section{Value of $\alpha_{s}(\boldsymbol{\mu})$}

In this work we use two classes of data for fixing $\alpha_{s}(\mu)$ and employ both procedures, (i) and (ii), described above; both procedures (i) and (ii) are used when $G_{A}(p)$ is obtained from functional methods, while (ii) is applied when $G_{A}(p)$ is taken from the lattice. For both classes of data, and for very different renormalization scales $[\mu=4.3$, $40 \mathrm{GeV}$ ], we will produce results for the quark propagator and the pion decay constant that agree within our estimated systematic error; this coincidence, in turn, constitutes a nontrivial check of the systematic errors. We next describe the determination of $\alpha_{s}(\mu)$ for both cases.

Functional datasets: Here we employ procedure (i). The functional input for $G_{A}(p)$ is provided by fRG [58] and SDE $[60,61]$ data, renormalized at $\mu=40 \mathrm{GeV}$. Note that the respective SDE and fRG relations for correlation functions are expanded about their $N_{f}=2$ counterparts, computed within the fRG [81]. The respective datasets also include $\alpha_{q \bar{q} A}(\bar{p})$, thus providing directly $\alpha_{s}(\mu)$ at $\mu=40 \mathrm{GeV}$; this allows us to minimize the difference between input and output $\alpha_{q \bar{q} A}(\bar{p})$, for $\bar{p} \gtrsim p_{\text {pert }}$.

Functional and lattice datasets: For both functional and lattice input data, we employ procedure (ii). We use the lattice data for $G_{A}(p)$ from $[31,83,84]$, and, in line with our arguments, we chose the maximal lattice momentum available for our renormalization scale, namely $\mu=4.3 \mathrm{GeV}$. The STI function $L_{1}$ in (5.4) is computed based on the quenched computation in [68] (Fig. 17, fourth panel), properly accounting for unquenching effects. Then, we minimize the difference between the left- and right-hand sides in (5.4). The same procedure is applied to the functional dataset at both $\mu=4.3 \mathrm{GeV}$ and $\mu=40 \mathrm{GeV}$.

Both procedures are now applied at two rather disparate renormalization scales $\mu$, namely $\mu_{1}=4.3 \mathrm{GeV}$ and $\mu_{2}=40 \mathrm{GeV}$, for which we obtain the values

$\alpha_{s}(4.3 \mathrm{GeV})=0.433, \quad \alpha_{s}(40 \mathrm{GeV})=0.166$,

which are fully compatible with earlier SDE and fRG considerations. The coincidence of the couplings for both procedures (i) and (ii) is a further nontrivial consistency check of the present RG scheme and corroborates the correct implementation of the present MOM-type scheme in the present SDE approach (for more details see Sec. A). The value of the coupling at the scale $M_{Z}$ obtained in this setup is $\alpha_{s}\left(M_{Z}\right)=0.14$. The deviation from the standard value $\alpha_{s, \text { phys }}\left(M_{Z}\right) \approx 0.12$ is due to the presence of only three active flavors in our analysis and the modifications in the present scheme in comparison to the standard MOM scheme. 


\section{CURRENT QUARK MASSES AND BENCHMARK PREDICTIONS}

In this section we determine the fundamental parameters of QCD, the current-quark masses $m_{q}=\left(m_{l}, m_{s}\right)$, where we have assumed isospin symmetry with identical up and down quark current masses: $m_{u / d}=m_{l}$. In addition, we provide results for a benchmark observable, namely the light chiral condensate, $\Delta_{l}=-\langle\bar{l}(x) l(x)\rangle$, which allows us to evaluate the veracity of the present approximations. In particular, we find that our $\Delta_{l}$ is in excellent quantitative agreement with the most recent lattice estimates reported in [99].

The current quark masses $m_{q}(\mu)$ at a given $\mu$ are fixed from the physical pion mass, $m_{\pi}$, and the ratio of strange and light current quark masses, $m_{s}(\mu) / m_{l}(\mu)$. This procedure has been used both in $[58,60,61,77,81]$ (see also the reviews $[4,13,15,100]$ ) and in lattice simulations (see, e.g., the compilation in [99]).

Note that, due to the identical (one-loop) RG running of all $m_{q}(\mu)$, the mass ratio $m_{s}(\mu) / m_{l}(\mu)$ tends to a constant for asymptotically large $\mu$,

$$
\begin{aligned}
\lim _{\mu \rightarrow \infty} \frac{m_{s}(\mu)}{m_{l}(\mu)} & =\frac{m_{s}}{m_{l}}, \quad m_{q}(\mu) \rightarrow \frac{m_{q}}{\left[\ln \left(\mu / \Lambda_{\mathrm{QCD}}\right)\right]^{\gamma_{m}}}, \\
\text { with } \quad \gamma_{m} & =\frac{12}{33-2 N_{f}} .
\end{aligned}
$$

In the present work we use $\mu=40 \mathrm{GeV}$ and compare the results to those obtained with a considerably lower $\mu=4.3 \mathrm{GeV}$, whose choice was dictated by the restricted momentum range of the lattice input.

For $\mu=40 \mathrm{GeV}$, the $m_{q}(\mu)$ will be determined using the values

$\left(m_{q}(\mu), \mu=40 \mathrm{GeV}\right): m_{\pi}=138 \mathrm{MeV}$, and $\frac{m_{s}}{m_{l}}=27$.

\section{A. Pagels-Stokar formula and Gell-Mann-Oakes-Renner relation}

Ideally, the pion mass, $m_{\pi}$, and decay constant, $f_{\pi}$, should be determined from the on-shell properties of the BS wave function of the pion. Instead, in the present work we employ standard Euclidean approximations for them, given by the Pagels-Stokar (PS) formula for $f_{\pi}$ and the GellMann-Oakes-Renner (GMOR) relation for $m_{\pi}[(6.3)$ and (6.9), respectively]. The GMOR relation is correct up to order $\mathcal{O}\left(m_{l}^{2}\right)$ within an expansion about the chiral limit, while the PS formula is known to underestimate $f_{\pi}$ by $\lesssim 10 \%$ (see, e.g., $[101,102]$ and the reviews $[4,15,103]$ ). We emphasize that this low value does not undermine the precision of our analysis, given that $f_{\pi}$ is a derived quantity that does not feed back into the SDEs.
The PS formula reads

$$
f_{\pi}^{(\mathrm{PS})}=\frac{4 N_{c}}{N_{\pi}} \int_{p} \frac{\bar{Z}_{2}}{Z_{q}(p)} \frac{\bar{M}_{q}(p)}{\left[p^{2}+M_{q}^{2}(p)\right]^{2}}\left[M_{l}(p)-\frac{p^{2}}{2} M_{q}^{\prime}(p)\right],
$$

with $M_{q}^{\prime}(p)=\partial_{p^{2}} M_{q}(p)$, and the subtracted mass function $\bar{M}_{q}(p)$,

$$
\bar{M}_{q}(p):=M_{q}(p)-m_{q} \frac{\partial M_{q}(p)}{\partial m_{q}} \text {, with } \lim _{p \rightarrow \infty} p^{2} \bar{M}_{q}(p)=0
$$

Note that Eq. (6.4) applies to both $m_{l}$ or $m_{s}$. The constant $\bar{Z}_{2}$ is linked to the quark dressing function $Z_{q}(p)$. Specifically, the derivation of (6.4) from the pion BetheSalpeter equation (BSE) prompts the identification $\bar{Z}_{2}=Z_{q}\left(p_{\pi}\right)$, where $p_{\pi}^{2}=-m_{\pi}^{2}$; in the chiral limit, $p_{\pi}^{2}=0$, and we will use $\bar{Z}_{2}=Z_{l}(0)$. Note the difference to the standard PS formula based on MOM, where $\bar{Z}_{2}=Z_{2}$.

The normalization $N_{\pi}$ of the pion wave function is given by

$$
N_{\pi}=\frac{1}{2}\left[f_{\pi}+\sqrt{f_{\pi}^{2}+8 N_{c} I_{l}}\right],
$$

with

$I_{q}:=\int_{p} \frac{\bar{M}_{q}^{2}(p)\left[p^{2} Z_{q}(p) Z_{q}^{\prime \prime}(p)+2 Z_{q}(p) Z_{q}^{\prime}(p)-p^{2} Z_{q}^{\prime \prime}(p)\right]}{Z_{q}^{2}(p)\left[p^{2}+M_{q}^{2}(p)\right]}$,

where the abbreviations $Z^{\prime}(p)=\partial_{p^{2}} Z(p)$ and $Z^{\prime \prime}(p)=$ $\partial_{p^{2}} Z^{\prime}(p)$ have been used. Note that, due to the asymptotic behavior of $\bar{M}_{q}(p)$ stated in (6.4), the integral is finite. The above expressions for $N_{\pi}$ are approximate; a more complete treatment requires all components of the pion wave function and will be considered elsewhere.

It is well-known that, in the chiral limit, we must have $I_{q, \chi}=0$ and $N_{\pi}=f_{\pi}$ [104]. However, our approximations deviate slightly from this result, furnishing a $I_{q, \chi}$ which fails to vanish by an amount that induces a $3 \%$ discrepancy between $N_{\pi}$ and $f_{\pi}$. We effectively account for this small error by setting

$$
I_{q} \rightarrow I_{q}-I_{q, \chi},
$$

thus compensating, in a simple way, for the contributions of the omitted form factors. The numerical impact of this adjustment will be discussed in Sec. VIC; see (6.18). 

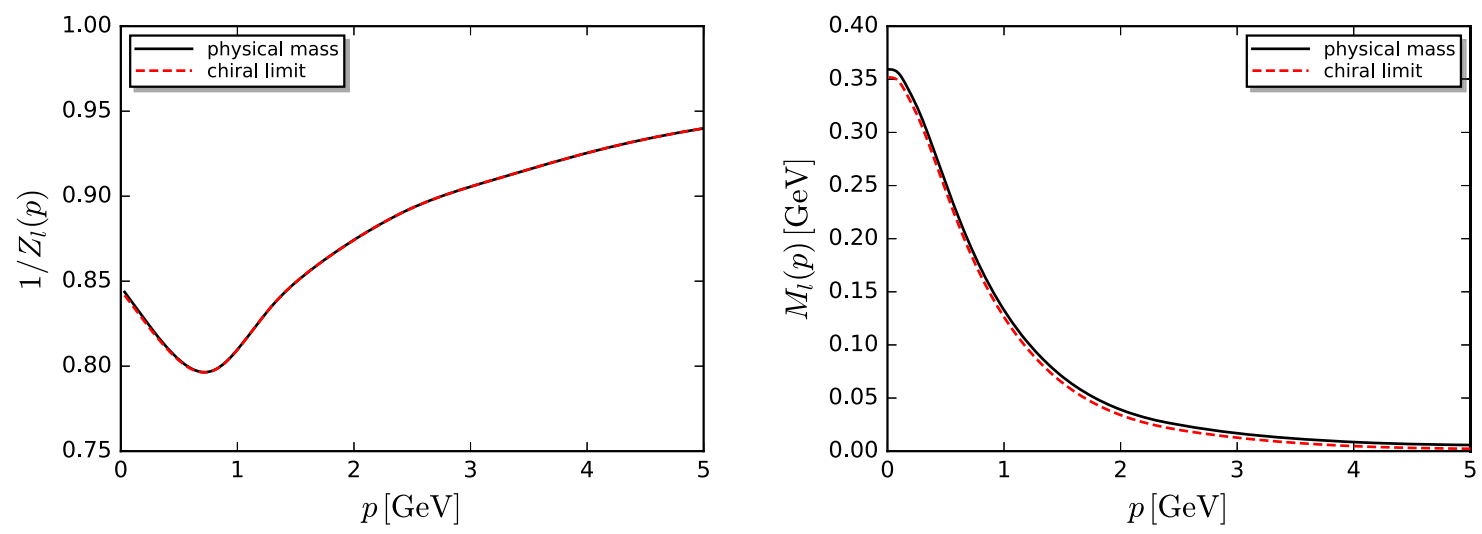

FIG. 4. Quark dressing, $1 / Z_{q}\left(p^{2}\right)$, and mass function, $M_{q}\left(p^{2}\right)$, in the chiral limit and for physical quark masses.

In the chiral limit we arrive at

$$
f_{\pi, \chi}^{(\mathrm{PS})}=84.9 \mathrm{MeV},
$$

in comparison to the FLAG estimate of $f_{\pi, \chi}^{\text {lat }}=86.7 \mathrm{MeV}$ and $f_{\pi}^{\text {lat }}=92.1(0.6) \mathrm{MeV}$ for physical quark masses.

Turning to the GMOR relation, we have

$m_{\pi}^{2}=\frac{2 m_{l}}{f_{\pi}^{2}} \Delta_{l}+\mathcal{O}\left(m_{l}^{2}\right), \quad$ with $\quad \Delta_{l}=-\langle\bar{u} u\rangle=-\langle\bar{d} d\rangle$

where $m_{l}$ is a $\mu$-independent current quark mass and $\Delta_{l}$ denotes the finite and $\mu$-independent light quark condensate; for a concise discussion of its RG properties, see [105].

Since in our calculations enter the $\mu$-dependent current quark masses, $m_{l}(\mu)$, rather than $m_{l}$, for the purposes of the present work, we find it advantageous to capitalize on the property

$$
m_{l} \Delta_{l}=m_{l}(\mu) \Delta_{l}(\mu),
$$

and recast (6.9) in the form

$$
m_{\pi}^{2}=\frac{2 m_{l}(\mu)}{f_{\pi}^{2}} \Delta_{l}(\mu)
$$

Evidently, in order to extract from (6.11) the value of $m_{l}(\mu)$, one requires knowledge of $f_{\pi}$ and $\Delta_{l}(\mu)$. Given the aforementioned shortcomings of the PS formula, we will simply use the physical value of $f_{\pi}$ as input in (6.9). The details of $\Delta_{l}(\mu)$ will be discussed separately below.

\section{B. Light chiral condensate}

The benchmark observable for our computation is the $\mu$-independent $\Delta_{l}$, whose chiral limit value, $\Delta_{l, \chi}$, can be extracted from the UV behavior of the corresponding constituent quark mass $M_{l, \chi}(p)$ [105],

$$
\lim _{p \rightarrow \infty} M_{l, \chi}(p)=\frac{2 \pi^{2} \gamma_{m}}{3} \frac{\Delta_{l, \chi}}{p^{2}\left[\ln \left(p / \Lambda_{\mathrm{QCD}}\right)\right]^{1-\gamma_{m}}},
$$

with $\gamma_{m}$ defined in (6.1).

Of course, as mentioned above, the quantity that we will employ in (6.11) is rather $\Delta_{l}(\mu)$. The latter is usually computed in lattice simulations at a given scale $\mu_{\text {lat }}$, which is typically far lower than the one used in the present SDE approach; for more details and an overview of the respective lattice results see [99]. For sufficiently large $\mu, \Delta_{l}$ and $\Delta_{l}(\mu)$ are related by

$$
\Delta_{l}(\mu)=\Delta_{l}\left[\ln \left(\mu / \Lambda_{\mathrm{QCD}}\right)\right]^{\gamma_{m}} .
$$

With $m_{l}(\mu)$ defined in (6.1) and (6.13), the combination $m_{l}(\mu) \Delta_{l}(\mu)$ is RG invariant, as stated in (6.10).

Combining the above relations with our SDE results for the quark propagator we arrive at our first nontrivial prediction. We use our results for $M_{l}(p)$ and $1 / Z_{l}(p)$ in the chiral limit, shown in Fig. 4, which are necessary for our analysis; a complete discussion is provided in Sec. VII. Specifically, the value of $\Delta_{l, \chi}$ is given by

$$
\Delta_{l, \chi}=(245 \mathrm{MeV})^{3} \text {, }
$$

where $m_{s}$ is kept fixed and $m_{l} \rightarrow 0$.

To fully appreciate the above prediction, we emphasize that, as can be shown analytically by identifying the diagrams included in each perturbative order, the current approach encodes the full two-loop running of $M_{l}(p)$ and is numerically consistent with the full three-loop running; for a more detailed discussion, see Sec. VII.

We next combine (6.14) with (6.13) to obtain our prediction for $\Delta_{l, \chi}(\mu)$, and we compare it with the FLAG result of [99], obtained from different lattice groups. In these simulations, the renormalization scale is $\mu_{\text {lat }}=2 \mathrm{GeV}$, and the estimate for the QCD scale is $\Lambda_{\mathrm{QCD}}^{\text {lat }}=343(12) \mathrm{MeV}$ in the $\overline{\mathrm{MS}}$ scheme; instead, in the present SDE computation we have $\Lambda_{\mathrm{QCD}}=293 \mathrm{MeV}$. From (6.13) and (6.14), this leads us to 


$$
\begin{aligned}
\Delta_{l, \chi}\left(\mu_{\text {lat }}\right) & =(270 \mathrm{MeV})^{3}, \\
{\left[\Delta_{l, \chi}\left(\mu_{\text {lat }}\right)\right]_{\text {FLAG }} } & =(272(5) \mathrm{MeV})^{3} .
\end{aligned}
$$

Taking into account the subtleties in the conversion and application of RG scales, the agreement between these two values is rather impressive, providing nontrivial support for the quantitative reliability of the present approximation. We emphasize that the prediction (6.14), and hence (6.15), has been obtained without any phenomenological input on the strength of chiral symmetry breaking.

Finally, $\Delta_{l, \chi}$ can be used for the determination of the physical $\left(m_{q} \neq 0\right)$ chiral condensate $\Delta_{l}$ through

$$
\begin{aligned}
\Delta_{l} & =\Delta_{l, \chi}+\left(\Delta_{l}-\Delta_{l, \chi}\right) \\
& =\Delta_{l, \chi}+\int_{p}\left(\frac{Z_{2}}{Z_{q}(p)} \frac{\bar{M}_{l}(p)}{p^{2}+M_{l}^{2}(p)}-\frac{Z_{2}}{Z_{q, \chi}(p)} \frac{M_{l, \chi}(p)}{p^{2}+M_{l, \chi}^{2}(p)}\right),
\end{aligned}
$$

where the integral is simply the difference of the loop expressions for the chiral condensates and is finite. In (6.16) we have already set the multiplicative renormalization factors to unity, according to the procedure described in Sec. VA. The calculated value for $\Delta_{l}$ is reported in the following subsection.

\section{Determination of the current quark masses}

With the groundwork laid in Secs. VI A and VI B, we now determine the current quark masses. Using (6.2), (6.3), (6.11), (6.16), and a pion decay constant $f_{\pi}=92.1 \mathrm{MeV}$, we arrive at the current quark masses $m_{q}$ at $\mu=40 \mathrm{GeV}$,

$$
\begin{aligned}
& m_{q}(\mu)=2.7 \mathrm{MeV}, \quad m_{s}=73 \mathrm{MeV}, \\
& \text { with } \quad m_{\pi}=138 \mathrm{MeV},
\end{aligned}
$$

and the predictions

$$
\begin{aligned}
\Delta_{l} & =(300 \mathrm{MeV})^{3}, \quad M_{q}(0)=351 \mathrm{MeV}, \\
f_{\pi}^{(\mathrm{PS})} & =87.6 \mathrm{MeV} .
\end{aligned}
$$

From (6.17b) we deduce that $f_{\pi}^{(\mathrm{PS})}$ has an error of $5 \%$, which is well within our conservative estimate for the systematic error in the range of $10 \%$. Note that this error estimate also extends to the GMOR relation, and hence our quark masses share this $10 \%$ uncertainty. We emphasize that this error is not inherent to our SDE computation, but affects deduced observables. Indeed, our benchmark result (6.15) for the chiral condensate in the chiral limit agrees with the lattice results within the statistical error of the latter (less than $1 \%$ deviation).

For completeness we also report the result for $f_{\pi}^{(\mathrm{PS})}$ without the correction to $N_{\pi}$ implemented by (6.7); we have

$$
\left[f_{\pi}^{(\mathrm{PS})}\right]_{I_{q, \chi} \neq 0}=86.9 \mathrm{MeV}
$$

in very good agreement with the $f_{\pi}^{(\mathrm{PS})}$ in (6.17b).

All results presented thus far have been obtained using the fRG data for the gluon propagator, renormalized at $\mu=40 \mathrm{GeV}$, as input in the SDEs. In order to illustrate that our predictions are essentially independent of the input propagator, we also report the results obtained when a fit to the gluon lattice data is employed, which has the fRG perturbative behavior built in it. Note also that the fRG and lattice data differ in the deep infrared (see Fig. 3), as commented in Sec. V B. With this particular input we arrive at

$$
\begin{aligned}
m_{q}(\mu) & =2.7 \mathrm{MeV}, \quad m_{s}=73 \mathrm{MeV}, \quad \text { with } \\
m_{\pi} & =138 \mathrm{MeV},
\end{aligned}
$$

and the predictions

$$
\begin{aligned}
\Delta_{l} & =(301 \mathrm{MeV})^{3}, \quad M_{q}(0)=350 \mathrm{MeV}, \\
f_{\pi}^{(\mathrm{PS})} & =88.1 \mathrm{MeV} .
\end{aligned}
$$

In addition, the results for chiral condensate in the chiral limit, $\Delta_{l, \chi}=(246 \mathrm{MeV})^{3}$ and $\Delta_{l, \chi}\left(\mu_{\text {lat }}\right)=(271 \mathrm{MeV})^{3}$ are in quantitative agreement with (6.14) and (6.15), obtained for $\mu=40 \mathrm{GeV}$. This coincidence and the comparison of (6.19) with (6.17) show explicitly that, within our estimated systematic error, the behavior of the gluon propagator in the deep infrared does not affect the observables considered here.

This concludes the discussion of the determination of the current quark masses. In summary, we have shown that the present SDE approach allows for a quantitatively reliable computation of the constituent quark mass function $M_{q}(p)$, and hence the chiral condensate in (6.15), without any phenomenological input.

\section{NUMERICAL RESULTS}

In this section we present and discuss the central results of our numerical analysis, focusing mainly on the general features displayed by $Z_{q}, M_{q}$, and the $\lambda_{i}$. The stability of these results under variations of the UV cutoff, the gluon inputs, and the RG scale will be addressed in Sec. VIII, while the implementation of reliable simplifications will be analyzed in Sec. IX.

Within the setup described in Secs. IV-VI, the SDE system of the quark propagator and quark-gluon vertex is solved iteratively. To that end, it is convenient to use as the starting point of the iteration input data for quark and gluon propagators and the respective $\mathrm{BC}$ vertex, or, when the fRG-DSE gluon is utilized [60,61], the respective quarkgluon vertex data. Moreover, the dressings of the three dominant tensor structures, $\lambda_{1,4,7}$, are fed back into their 


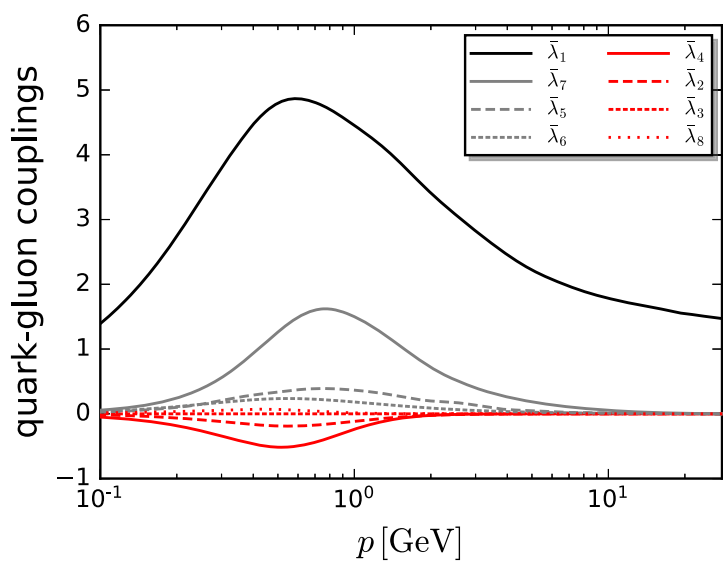

(a)

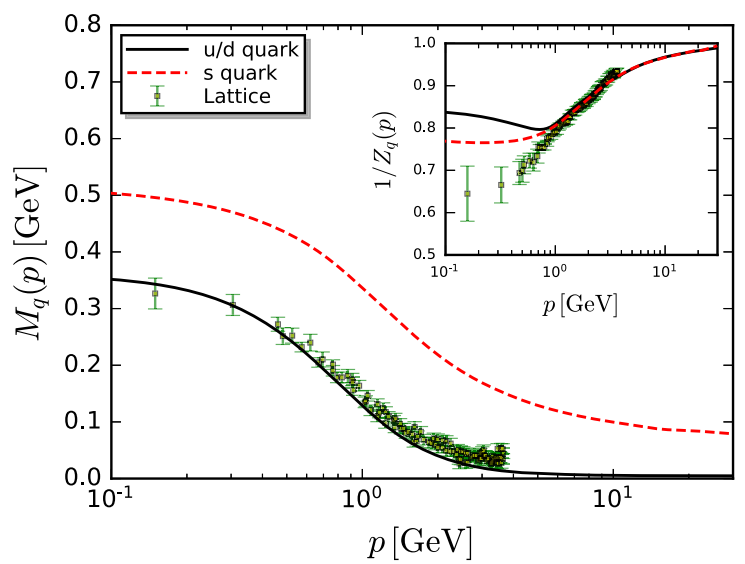

(b)

FIG. 5. Numerical results for the coupled system of SDEs for quark-gluon vertex (light quark, left panel), and the light and strange quark propagators (right panel). (a) Quark-gluon couplings $\bar{\lambda}_{i}(p)$, (7.4). Black: classical tensor structure, (2.6). Grey: chirally symmetric nonclassical tensor structures. Red: chiral-symmetry breaking tensor structures. (b) Quark mass functions $M_{q}(p)$ and propagator dressings $1 / Z_{q}(p)$ (inset) for $q=l, s$, see (2.7). Lattice results from [106]: light quark mass function $M_{l}(p)$ and dressing $1 / Z_{l}(p)$.

own coupled SDEs and those of the other vertex dressings; instead, the remaining $\lambda_{2,3,5,6,8}$ are only used in the quark gap equation. We have checked that this procedure affects the key quantities only very marginally: when the subdominant dressings are fed back into the quark-gluon SDE, the numerical changes induced lie comfortably within the estimated error bars of our method.

The algorithm we employ for the numerical integrations is the standard Gauss-Legendre quadrature. To that end, we pass to spherical coordinates, with the loop measure given by

$$
d^{4} q=\frac{1}{2} q^{2} \sin ^{2} \theta \sin \phi d q^{2} d \theta d \phi d \psi,
$$

where $q^{2} \in[0, \infty), \theta \in[0, \pi], \phi \in[0, \pi]$, and $\psi \in[0,2 \pi]$. The azimuthal angle $\psi$ may be integrated trivially.

We next introduce the parametrization $q^{2}=\Lambda \kappa(\Lambda / \kappa)^{y}$, where $\kappa$ and $\Lambda$ denote the infrared and UV cutoffs, respectively. The new integration variable $y$ is related to $q^{2}$ by $y=\ln \left(q^{2} / \Lambda \kappa\right) / \ln (\Lambda / \kappa)$; evidently, $y \in[-1,1]$. In addition, the angular integrals are written in terms of the new integration variables $z=\cos \theta$ and $z^{\prime}=\cos \phi$, again with $z, z^{\prime} \in[-1,1]$. These changes of variables facilitate the use of the Gauss-Legendre quadrature, according to which an integral of a function $f(x)$, with $x \in[-1,1]$, is written as

$$
\int_{-1}^{1} d x f(x) \approx \sum_{i=1}^{n} w_{i} f\left(x_{i}\right)
$$

where the nodes $x_{i}$ and weights $w_{i}$ are uniquely determined by requiring that (7.2) becomes exact for all polynomials of degree less than $2 n$. Specifically, one may show that the $x_{i}$ are the roots of the $n$th Legendre polynomial, $P_{n}(x)$, and the weights are given by the formula

$$
w_{i}=\frac{2}{\left(1-x_{i}^{2}\right)\left[P_{n}^{\prime}\left(x_{i}\right)\right]^{2}} .
$$

For the actual calculation, we set $n=40$ for the integration over $y$ and $n=20$ for the integrations over $z$ and $z^{\prime}$.

Finally, once the integrations have been carried out, one is left with a sizable system of coupled nonlinear algebraic equations, which is solved by means of Broyden's method, with the numerical precision set at $10^{-5}$.

As discussed in Sec. VIII, the RG scale $\mu$ should be taken as large (i.e., as "perturbative") as possible; therefore, we choose $\mu=40 \mathrm{GeV}$. However, the lattice data for the gluon propagator are limited by $p \lesssim 5 \mathrm{GeV}$ and have to be extended by a perturbative fit; instead, the functional data for the gluon propagator cover the full momentum range of interest. Therefore, we employ the functional data from $[58,60,61]$ as our input for the present computation, while the lattice data from $[31,83,84]$ are used as benchmark results for the low momentum regime; see Fig. 3.

The above setup represents our best approximation and allows us to compute the quark-gluon vertex as well as the quark propagator without any phenomenological input; in particular, the only parameters to be fixed are the fundamental parameters of QCD, namely the current quark masses at the RG scale $\mu$; see Sec. VI.

Our main results are summarized in Fig. 5. In particular, in Fig. 5(a) we show all dressings of the quark-gluon vertex, while in Fig. 5(b) we display the quark mass functions, $M_{q}(p)$, and the quark wave function renormalizations $1 / Z_{q}(p)$ (inset) for $q=l, s$. Note that, in order to best expose the physical relevance of the different tensor 


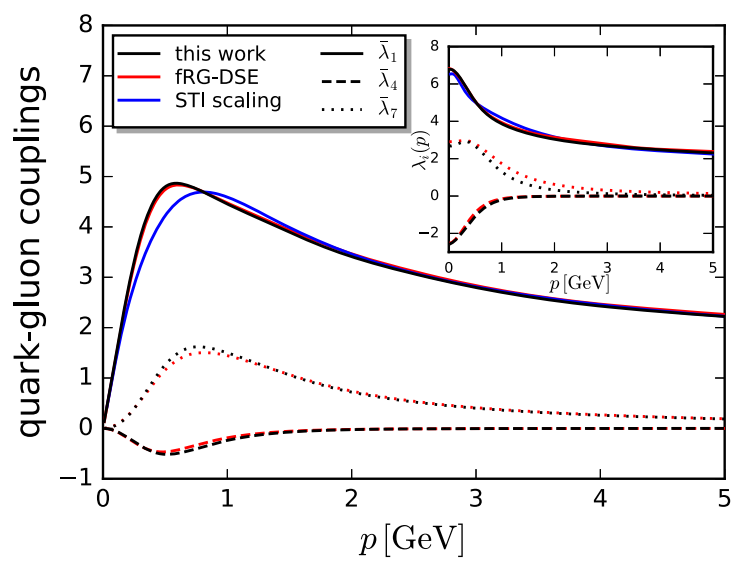

(a)

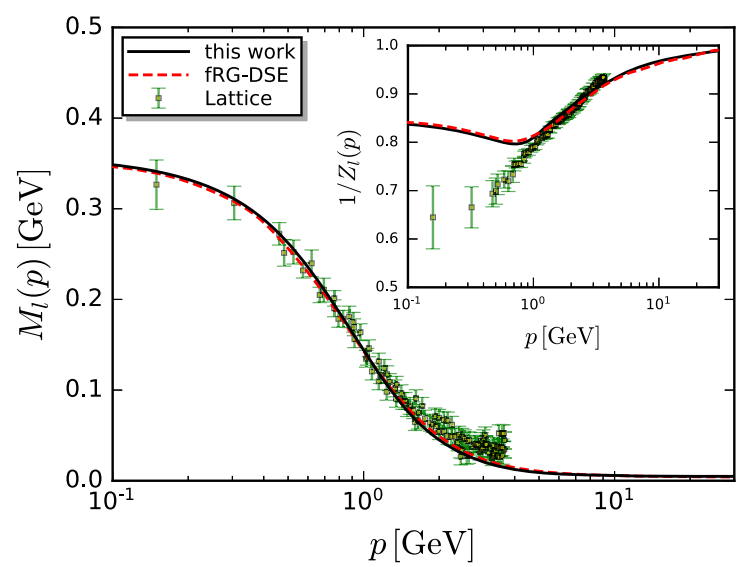

(b)

FIG. 6. Full results for dominant quark-gluon couplings $\bar{\lambda}_{1,4,7}$ (this work, black) from the present work in comparison to full results from [61] (fRG-DSE, red), lattice results (Lattice, green), and the approximation STI scaling: $\lambda_{1,2,6}$ from STI, and $\lambda_{4,5,6,7}$ from scaling relations (see discussion at the end of Sec. IX and [60,81]). The $\lambda_{i}$ are dressings of tensor structures in Eq. (4.11a) and measured in $\lambda_{1}$, $\lambda_{4}\left[(\mathrm{GeV})^{-1}\right]$ and $\lambda_{7}\left[(\mathrm{GeV})^{-2}\right]$. (a) Dominant quark gluon couplings $\lambda_{1,4,7}$ : full solution SDE (this work, black), full solution [60] (fRGDSE, red), couplings with STI, $\bar{\lambda}_{1}$, and scaling relations, $\bar{\lambda}_{4,7}$ (STI-scaling, blue). (b) Light quark mass function $M_{l}(p)$ and dressing function $1 / Z_{l}(p)$ (inset): full solution (this work, full black), full solution [60] (fRG-DSE, dashed red), lattice results [106] (Lattice, green).

structures and the corresponding dressings, we introduce dimensionless couplings, $\bar{\lambda}_{i}(\bar{p})$, with $i=1, \ldots, 8$ (see, e.g., [77]); specifically, we concentrate on the symmetric point $\bar{p}$ and define

$\begin{aligned} \bar{\lambda}_{i}(\bar{p}) & =\frac{\bar{p}^{n_{i}} \lambda_{i}(\bar{p})}{Z_{q}(\bar{p}) Z_{A}^{1 / 2}(\bar{p})}, \quad \text { with } \quad n_{1}=0, \\ n_{2,3,4} & =2, \quad n_{5,6,7}=2, \quad n_{8}=3 .\end{aligned}$

In (7.4), the multiplication of $\lambda_{i}$ by $\bar{p}^{n_{i}}$ renders the $\bar{\lambda}_{i}$ dimensionless, and the division by the wave function renormalisations leaves us with the respective eight running couplings.

For all quarks $q=l, s$, the products $1 /(4 \pi) \bar{\lambda}_{i}(\bar{p}) \bar{\lambda}_{j}(\bar{p})$ can be interpreted as the interaction strength of a onegluon exchange between corresponding quark currents. For example, $\alpha_{q \bar{q} A}(\bar{p})=1 /(4 \pi)\left[\bar{\lambda}_{1}(\bar{p})\right]^{2}$ [see (2.6)] simply measures the interaction strength or running coupling of a one-gluon exchange between the two quark currents $\bar{q}(t) \gamma_{\mu} q(p-t)$. Similarly, the combinations $1 /(4 \pi) \bar{\lambda}_{i}(\bar{p}) \bar{\lambda}_{j}(\bar{p})$ can be understood as the interaction strength of a one-gluon exchange between the respective tensor currents $\bar{q} \overline{\mathcal{T}}_{i} q$ and $\bar{q} \overline{\mathcal{T}}_{j} q$, with the dimensionless tensor structures $\overline{\mathcal{T}}_{i}=\mathcal{T}_{i} /\left(\bar{p}^{2}\right)^{n_{i}}$, with $i, j=1, \ldots, 8$. As in the case of the gluon dressing, one sees the prominent enhancement around $\bar{p} \approx 0.5-1 \mathrm{GeV}$, which is crucial for obtaining from the gap equation the correct amount of chiral symmetry breaking. Moreover, we note the clear suppression of all $\bar{\lambda}_{i}$ for $\bar{p} \rightarrow 0$.
In Fig. 6, we compare our results to those obtained through a combined setup [60,61] (fRG-DSE), where the relevant SDEs are expanded about the two-flavor QCD correlation functions of [81]. As we may infer from Fig. 6, the present SDE results are quantitatively consistent with those of $[60,61]$. This is an additional, highly relevant reliability check for the respective results of different but similar functional approaches. In our opinion, the confirmation of this quantitative agreement and further successful comparisons of this type provide important information about the respective systematic error. Together with apparent convergence of the results in functional approaches within a systematic approximation scheme, this finally will lead to a first principle functional approach to QCD.

The interpretation of the comparison of the dressing $1 / Z_{l}(p)$ from functional methods and the lattice is less clear. To begin with, the lattice result from [106] shows a rather steep slope at momenta $p \gtrsim 1 \mathrm{GeV}$. For $p \lesssim 0.5 \mathrm{GeV}$, it shows a rapidly rising statistical error. Clearly, it would be highly desirable to repeat the computation of the quark dressings with more recent sets of configurations, i.e., with $[31,83,84]$.

In turn, all functional studies, e.g., $[70,79,81]$, consistently show a smooth rise of the dressing $1 / Z_{l}(p=\mu)=1$ that is compatible with perturbation theory. For $p \lesssim 1 \mathrm{GeV}$, these studies show a nonmonotonic behavior, which cannot be identified within the statistical accuracy of the lattice data. This calls for more refined studies, as $1 / Z_{q}(p)$ carries significant systematic errors in this regime $p \lesssim 1 \mathrm{GeV}$. It is interesting to note that the existence and strength of this 

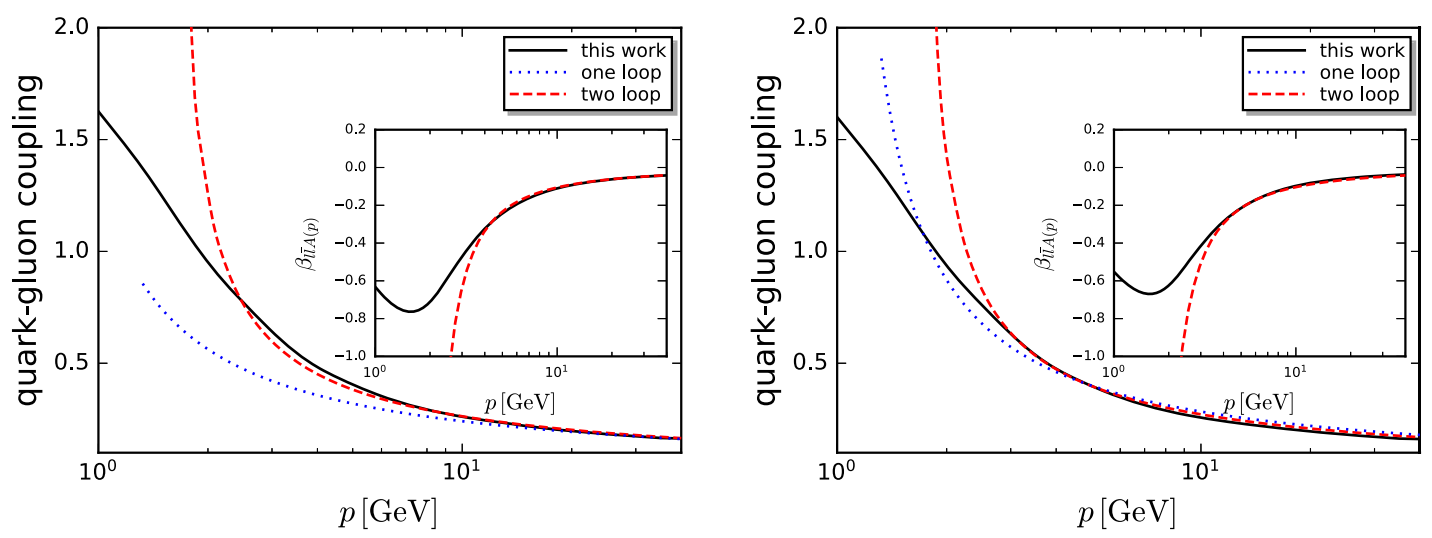

FIG. 7. Strong coupling $\alpha_{\overline{l l} A}(p)$ [see (2.6)] in comparison to the one- and two-loop counterparts, adjusted at the RG scale $\mu=40$ GeV (left panel) and $\mu=4.3 \mathrm{GeV}$ (right panel).

nonmonotonicity depends on the size of the current quark mass; see Fig. 5(b) for a comparison of $1 / Z_{l}$ and $1 / Z_{s}$ and [81] for a study of the $m_{l}$ dependence in two-flavor QCD. We hope to resolve this situation in a combined functionallattice study in the near future.

We emphasize that the present approximation includes analytically the full two-loop running of the quark gap equation. Hence, $M_{q}(p)$ and $1 / Z_{q}(p)$ are two-loop consistent, since the quark gap equation contains all tensors of the quark-gluon vertex. The SDE solution of the latter includes all one-loop diagrams, and hence, the numerical solution for the $\lambda_{i}(p, q)$ encompasses the full one-loop structure analytically. Furthermore, the input gluon data contain at least the full one-loop momentum dependence. Accordingly, all ingredients in the quark-gap equation carry at least their full one-loop momentum dependence, and hence, the solution is analytically two-loop consistent.

Of course, as already mentioned in Sec. IV, the vertex SDE employed (see Fig. 2) corresponds to the so-called "one-loop dressed" truncation, where vertices with no classical counterpart, such as the four-quark vertex [61], have been omitted from the skeleton expansion. Nonetheless, the contributions of such terms are very suppressed for perturbative momenta, as we have confirmed in the case of $\alpha_{\overline{l l} A}(p)=1 /(4 \pi) \bar{\lambda}_{1}^{2}(p)$. In Fig. 7 we depict our numerical result together with the analytic oneand two-loop strong couplings $\alpha_{s}^{(1 \text { loop })}(p)$ and $\alpha_{s}^{(2 \text { loop })}(p)$, renormalized at $\mu=40 \mathrm{GeV}$ and $\mu=4.3 \mathrm{GeV}$. Then, the respective values for $\Lambda_{\mathrm{QCD}}$ are chosen such that also the $\beta$-functions

$$
\beta_{\overline{l l} A}(p)=p \partial_{p} \alpha_{\bar{l} \bar{A}}(p),
$$

match at the renormalization scale $\mu$. The numerical results in the present work have been obtained with $\mu=40 \mathrm{GeV}$, which lies deep in the perturbative regime. While this choice reduces the systematic error originating from nonperturbative approximations to the SDEs, its successful implementation requires a particularly accurate treatment, in order to reliably connect the wide range of momenta between $\mu$ and the deep infrared.

As can be seen in Fig. 7 (inset left panel), our numerical results for $\beta_{\overline{l l} A}$ agree quantitatively with the respective twoloop results $\beta_{\alpha_{s}}^{(2 \text { loop })}$ for momenta $p \gtrsim 5 \mathrm{GeV}$, while the full coupling $\alpha_{\bar{l} \bar{A} A}$ and the two-loop coupling $\alpha_{s}^{(2 \text { loop })}$ agree even up to $p \approx 3 \mathrm{GeV}$.

Still, a careful analysis reveals that deviations in the pair $\left(\alpha_{s}, \beta_{\alpha_{s}}\right)$ start to become visible for $p \lesssim 10 \mathrm{GeV}$ (for a more detailed discussion see Appendix A 2); there, it is also shown that the two-loop prediction for $\Lambda_{\mathrm{QCD}}$ is stable for $p \gtrsim 10 \mathrm{GeV}$ and as required by $\mathrm{RG}$ consistency. For our RG scale of $\mu=40 \mathrm{GeV}$ we find $\Lambda_{\mathrm{QCD}}=1.42 \mathrm{GeV}$, within an estimated error of approximately $\pm 0.02 \mathrm{GeV}$. We conclude that the current setup is sufficiently accurate to allow for a self-consistent renormalization at a large perturbative $\mu$. However, the $\beta$-function $\beta_{\bar{l} \bar{l} A}$, which measures the momentum slope, starts to deviate from the two-loop result $\beta_{\alpha_{s}}^{(2 \text { loop })}$ in the momentum regime $p \in$ $(5-10) \mathrm{GeV}$ [see Fig. 7 (inset left panel)]. Consequently, in this regime, the required $\mu$ independence of $\Lambda_{\mathrm{QCD}}$ in a consistent RG scheme is lost gradually within a two-loop matching, leading to a slightly different $\Lambda_{\mathrm{QCD}}=1.49 \mathrm{GeV}$ for $\mu=4.3 \mathrm{GeV}$. For more details, see Appendix A 2, and in particular Fig. 14, where the transition regime between the perturbative and nonperturbative regimes is marked by a red band.

Below $p \approx 3 \mathrm{GeV}$, the strong coupling $\alpha_{\overline{l l} A}$ rapidly departs from the perturbative two-loop coupling, signaling the onset of nonperturbative physics. The lack of RG consistency with a two-loop matching for small RG scales is even more apparent within a one-loop matching. There, an adjustment of the one-loop coupling and its $\beta$-function at $\mu=40 \mathrm{GeV}$ leads to $\Lambda_{\mathrm{QCD}}=0.59 \mathrm{GeV}$, while at $\mu=$ $4.3 \mathrm{GeV}$ we are led to $\Lambda_{\mathrm{QCD}}=0.86 \mathrm{GeV}$. The lack of RG consistency at one loop is even more manifest in the fact 


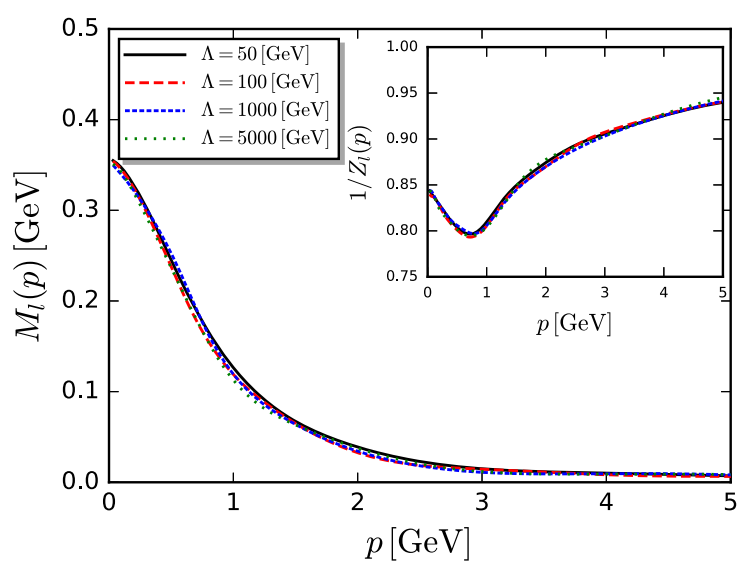

(a)

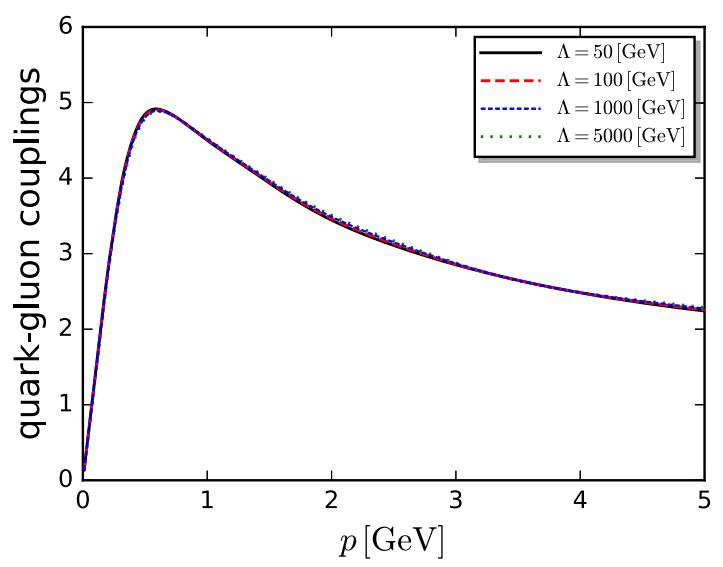

(b)

FIG. 8. Numerical results for the coupled system of SDEs for different UV cutoffs $\Lambda=50,100,1000,5000 \mathrm{GeV}$, based on the gluon input data [60,61] (fRG-DSE in Fig. 3). (a) Light quark mass function $M_{l}(p)$ and dressing $1 / Z_{l}(p)$ (inset) for different UV cutoffs. (b) Quark-gluon coupling $\bar{\lambda}_{1}(p)$ of the classical tensor structure for different UV cutoffs.

that a respective truncation clearly cannot bridge the wide momentum range between $p=40 \mathrm{GeV}$ and the nonperturbative infrared regime with $p \lesssim 5 \mathrm{GeV}$ [see Fig. 7 (left panel)].

Accordingly, our lower renormalization scale, $\mu=$ $4.3 \mathrm{GeV}$ is at the boundary between the perturbative and nonperturbative regimes. The two-loop coupling and the full $\alpha_{\bar{l} \bar{l} A}$ agree well for momenta $p \gtrsim 3 \mathrm{GeV}$, but, contrary to the case with $\mu=40 \mathrm{GeV}$, the $\beta$-function reveals deviations already in the perturbative regime [see Fig. 7 (inset right panel)].

This comparison carries an important message for phenomenological applications: potential inaccuracies of the present method that thwart the reliable bridging of disparate momentum scales can be compensated by choosing a relatively small renormalization scale. Nonetheless, such a choice is limited by a minimal RG scale, $\mu \geq \mu_{\min }$, with $\mu_{\min } \approx 3 \mathrm{GeV}$; smaller $\mathrm{RG}$ scales push renormalization clearly into the nonperturbative regime, where the arguments invoked in Sec. VA for setting $Z_{i}=1$ do not apply.

Finally, we note that a fully two-loop consistent analysis would require the omitted diagrams in the quark-gluon SDE, as well as a two-loop consistent gluon input. Both tasks lie within the technical grasp of functional approaches; for a discussion concerning the gluon propagator, see [55,107].

In summary the agreement with the lattice as well as other functional methods is rather impressive, especially since no phenomenological infrared parameter is involved: the results presented here are obtained within a firstprinciple setup to $\mathrm{QCD}$, the only input being the fundamental parameters of QCD.

\section{STABILITY OF THE NUMERICAL RESULTS}

As we will see in detail in this section, the results obtained from our SDE analysis are particularly stable under variations of the UV cutoff that regulates the loop integrals, the choice of functional or lattice gluon inputs, and a vast change in the value of the RG scale.

\section{A. Varying the UV cutoff}

We have verified explicitly that our results are practically insensitive to variations momentum cutoff $\Lambda$ within the range $\Lambda=50 \mathrm{GeV}$ to $\Lambda=5000 \mathrm{GeV}$. Note that, while $\lambda_{1}$ displays a marginal (logarithmic) cutoff dependence, all remaining $\lambda_{i}$ are not subject to renormalization. Moreover, in the Landau gauge, the logarithmic running of $Z_{l}(p)$ vanishes at one loop, and only $M_{l}(p)$ shows a one-loop logarithmic running. Accordingly, in Fig. 8 we show the absence of cutoff dependence in $M_{l}(p), 1 / Z_{l}(p)$, and $\bar{\lambda}_{1}(p)$. Our results are especially stable, and, in particular, no $\log \Lambda$ dependence may be discerned.

We emphasize that the detection of such a logarithmic dependence in the present system is very difficult, due to its (Landau gauge) suppression in $Z_{l}$, and the decay of $M_{l}(p)$ for large momenta. This leaves us with $\bar{\lambda}_{1}(p)$, whose perturbative momentum dependence is fixed by the selfconsistent determination described in Sec. V D. Note that these properties, even though they complicate the detection of residual cutof dependences, are a welcome feature rather than a liability: the present setup reduces the sensitivity of the SDE system with respect to the subtleties of a nonperturbative numerical renormalization.

\section{B. Stability with respect to the gluon input data}

We proceed with the insensitivity with respect to the gluon input data, described in Sec. V B and depicted in Fig. 3. In Fig. 9 we compare the results obtained using as inputs: (a) the data from the fRG-DSE computation $[60,61]$ (fRG-DSE input); (b) the gluon propagator obtained with the fRG computation of [58] (fRG input); and (c) the fit to 


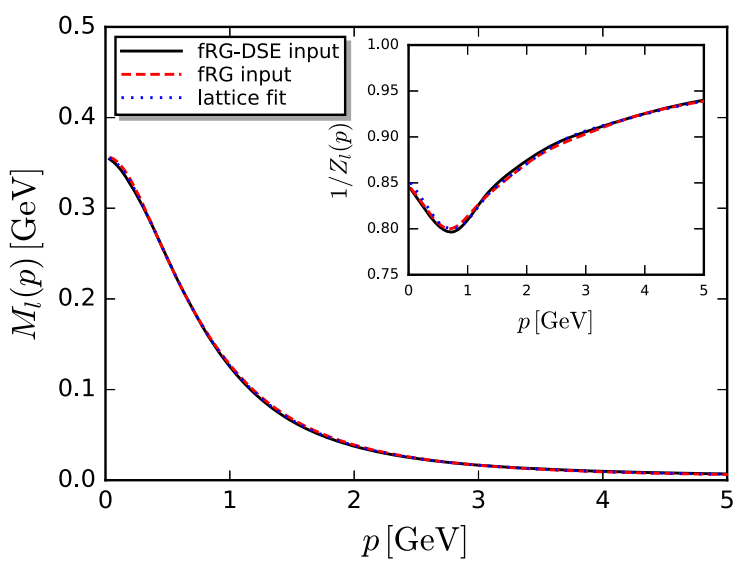

(a)

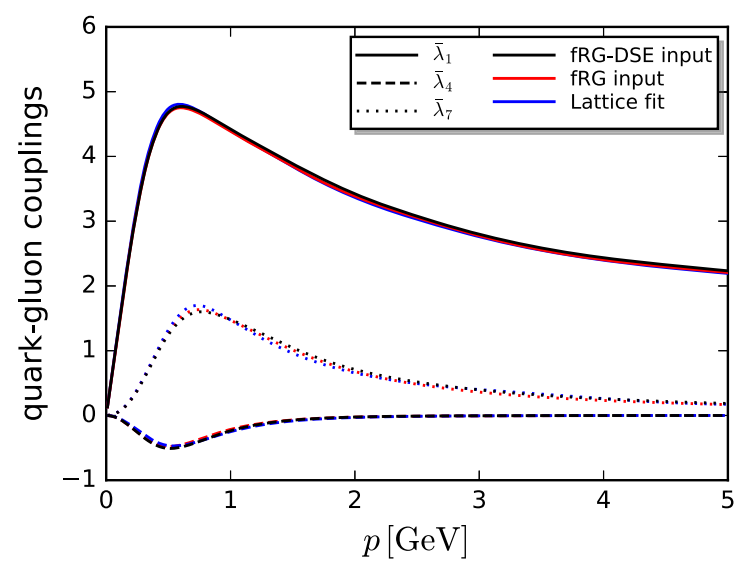

(b)

FIG. 9. Numerical results for the coupled system of SDEs with $\mu=40 \mathrm{GeV}$ for different gluon input data, Fig. 3 in Sec. V B: [60,61] (fRG-DSE), [58] (fRG), [31,83,84] (lattice fit). (a) Light quark mass function $M_{l}(p)$ and dressing $1 / Z_{l}(p)$ (inset) for different gluon input. (b) Dominant quark-gluon couplings $\bar{\lambda}_{1,4,7}(p)$ for different gluon input.

the lattice data of $[31,83,84]$, including an RG-consistent UV extrapolation (lattice fit). The respective results for $M_{l}(p)$ and $1 / Z_{l}(p)$ are shown in Fig. 9(a), while those for $\bar{\lambda}_{1,4,7}$ are in Fig. 9(b).

All results show an impressive quantitative agreement within the statistical and systematic errors. In particular, the difference in the infrared behavior between the lattice and the functional data used here (see Fig. 3) does not leave any significant trace on $M_{l}(p)$ and $1 / Z_{l}(p)$, as can be seen in Fig. 9(a). Accordingly, they do not influence our benchmark prediction for the chiral condensate, given in (6.15). Moreover, the same independence is seen at the level of the $\bar{\lambda}_{1,4,7}(p)$, displayed in Fig. 9(b). This lack of sensitivity to the infrared details of the input gluon propagators stems from the fact that the latter enter into four-dimensional momentum integrals, whose radial dependence, $p^{3}$, suppresses the deep infrared very effectively.

\section{Varying the RG scale $\mu$}

Finally we test the response of our results to changes in the RG scale $\mu$. In particular, we compare the results obtained when all relevant quantities have been renormalized at the two vastly different scales $\mu=40 \mathrm{GeV}$ and $\mu=4.3 \mathrm{GeV}$; in both cases we employ the fRG-DSE input.

Evidently, $M_{l}(p)$ and $\bar{\lambda}_{i}(p)$ are formally RG-invariant quantities, and, ideally, they should be $\mu$ independent; in practice, the amount of residual $\mu$ dependence displayed is an indication of the veracity of the approximations employed. The results shown in Fig. 10 demonstrate clearly that the $\mu$ dependence of these quantities lies well within the estimated error bars; in particular, the largest visible discrepancy, located at the peak of $\bar{\lambda}_{1}(p)$, is only $3.4 \%$.

On the other hand, the quantity $Z_{l}(p)$ is not RG invariant, depending explicitly on $\mu$, as can be seen in the inset of Fig. 10(a). However, multiplicative renormalization, when properly implemented, dictates that the curves renormalized at two different values of $\mu$, say $\mu_{1}$ and $\mu_{2}$, must be related by

$Z_{l}^{-1}\left(\mu_{2}, \mu_{1}\right) Z_{l}^{-1}\left(p, \mu_{2}\right)=Z_{l}^{-1}\left(p, \mu_{1}\right)$ with $\mu_{2}<\mu_{1}$.

The operation described in (8.1) rescales the "red-dashed" curve to the "blue-dotted" one in the aforementioned inset. Note that the rescaling factor is marked on the "black-solid" curve with a blue dot; its numerical value is 0.93 . Plainly, the coincidence achieved between original and rescaled curves is excellent, indicating that multiplicative renormalizability has been adequately implemented at the level of our dynamical equations.

\section{RELIABLE LOW-COST APPROXIMATIONS}

The numerical cost of the present work is rather modest: a full simulation with given gluon input data requires about 20 core minutes on an Intel i7 chip. However, if the system is extended by the gluon SDE in order to obtain a fully selfconsistent description, the numerical costs rises significantly. Moreover, for applications to hadron resonances (see, e.g., [15]), the SDE system has to be augmented by BSE, Faddeev equations, and four-body equations, depending on the resonances of interest. Finally, in the study of the QCD phase structure at finite temperature and density, a rest frame is singled out, leading to a further proliferation of tensorial structures. For all the above reasons, any approach that reduces the computational cost without compromising the veracity of the results, is potentially useful for the above applications.

In what follows we discuss simplified approximations of the treatment of the quark-gluon vertex that still lead to quantitatively reliable results. To that end, we analyze the numerical impact that the vertex dressings $\bar{\lambda}_{i}(p)$ have on the results of the quark dressings $M_{l}(p)$ and $1 / Z_{l}(p)$. 


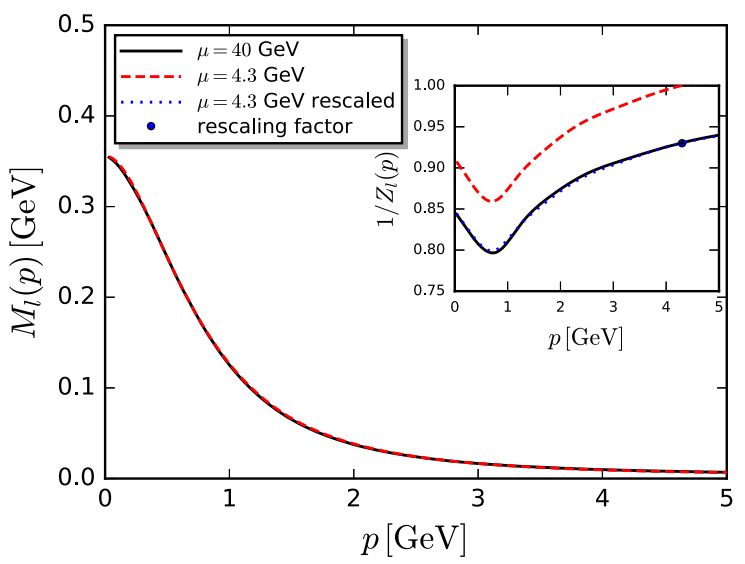

(a)

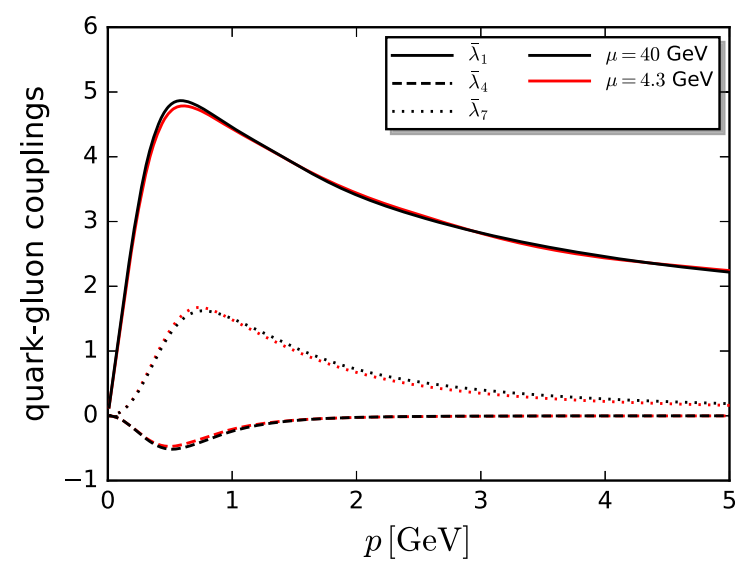

(b)

FIG. 10. The $\mu$ independence of $M_{l}(p)$ and $\bar{\lambda}_{1,4,7}(p)$, and multiplicative renormalizability of $Z_{l}(p)$. The RG scales differ by an order of magnitude: $\mu=40 \mathrm{GeV}$ and $\mu=4.3 \mathrm{GeV}$. (a) $M_{l}(p)$ and $Z_{l}(p)$ using the fRG-DSE input, for $\mu=40 \mathrm{GeV}$ (black-solid) and $\mu=$ $4.3 \mathrm{GeV}$ (red-dashed and blue-dashed (rescaled)). The blue dot indicates the rescaling factor. (b) Results for the dominant quark-gluon couplings $\bar{\lambda}_{1,4,7}$, obtained with the fRG-DSE input, and renormalised at $\mu=40 \mathrm{GeV}$ and $\mu=4.3 \mathrm{GeV}$.

To better appreciate this discussion, we have replotted the results for the $\bar{\lambda}_{i}(p)$, already shown in Fig. 5(a): in Fig. 11 we concentrate on the $\bar{\lambda}_{i}(p)$ in the low energy regime, i.e., for $p \lesssim 5 \mathrm{GeV}$. The $\bar{\lambda}_{i}(p)$ are separated in two groups, those with chiral symmetry preserving tensor structures, Fig. 11(a), and those with chiral symmetry breaking ones, Fig. 11(b).

The main outcome of these considerations may be summarized by stating that (i) the inclusions of $\lambda_{1,4,7}(p)$ are necessary and sufficient for approximating accurately the results of the full analysis, and (ii) $\lambda_{1}(p)$ may be reliably obtained from STI-based constructions, while $\lambda_{4,7}(p)$ from the scaling relations put forth in $[60,61,81]$.
Point (i) has been established by considering the relevant SDE approximations for the quark-gluon vertex that include $\lambda_{1}$ (which, obviously, cannot be omitted) and various subsets of $\left\{\lambda_{i_{1}}, \ldots, \lambda_{i_{n}}\right\}$. It is evident from Fig. 12 that the omission of either $\lambda_{4}$ or $\lambda_{7}$ (while keeping the rest) leads to sizable deviations from our best results for $M_{q}(p)$ and $1 / Z_{q}(p)$. Similarly, retaining only the special combination $\lambda_{1,4,7}(p)$ reproduces very accurately our best results for $M_{q}(p)$ and $1 / Z_{q}(p)$, as shown in Fig. 12.

Note also, that the hierarchy of form factors established in (i) is compatible with that of the corresponding couplings $\bar{\lambda}_{i}(p)$, whose relative size is shown in detail in Fig. 11 . As we can see there, $\bar{\lambda}_{1,4,7}(p)$ are indeed the largest

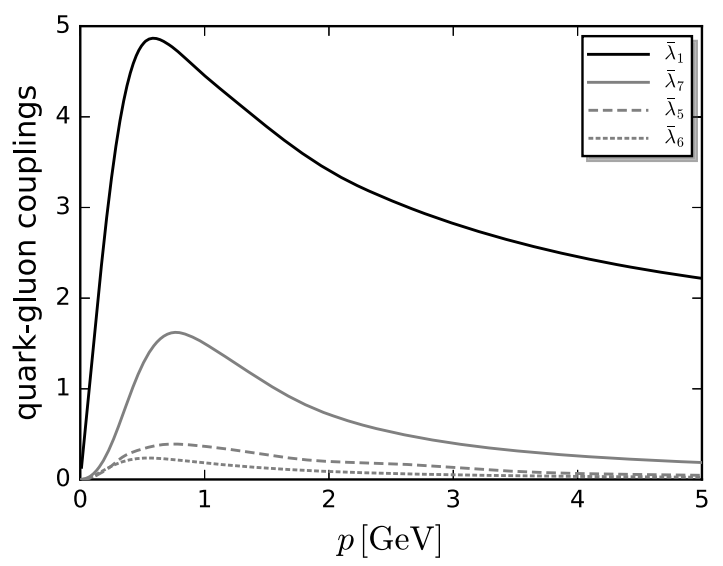

(a)

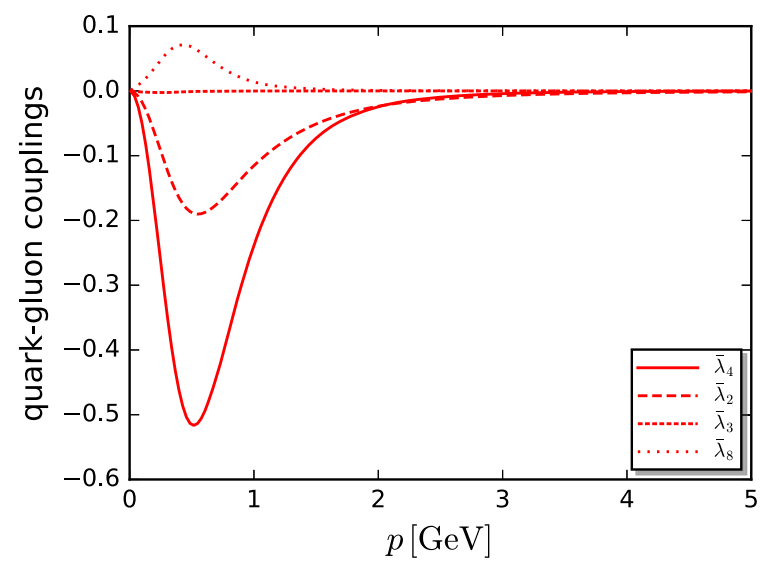

(b)

FIG. 11. Quark-gluon couplings $\bar{\lambda}_{i}(p)$, defined at the symmetric point; see (7.4). The ordering in the legends is reflecting their strength. (a) Quark-gluon couplings from chirally symmetric tensor structures $\mathcal{T}_{1,5,6,7}$. (b) Quark-gluon couplings from the chiralsymmetry-breaking tensor structures $\mathcal{T}_{2,3,4,8}$. 


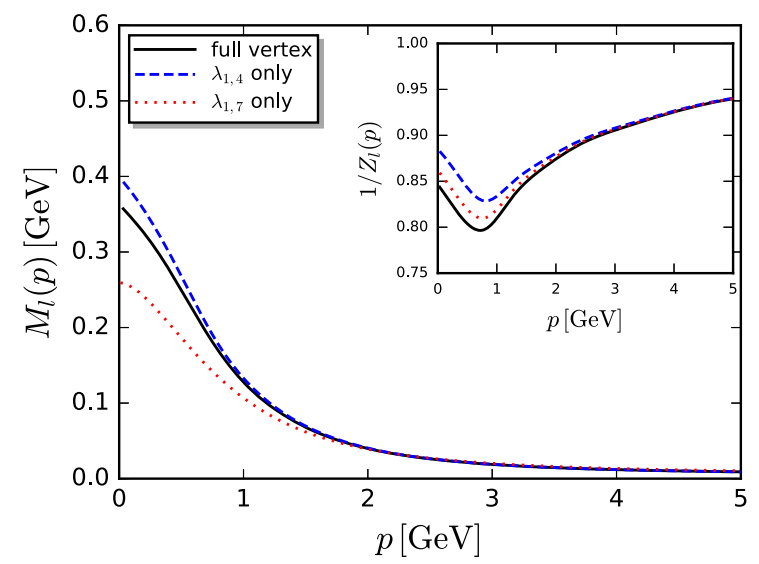

(a)

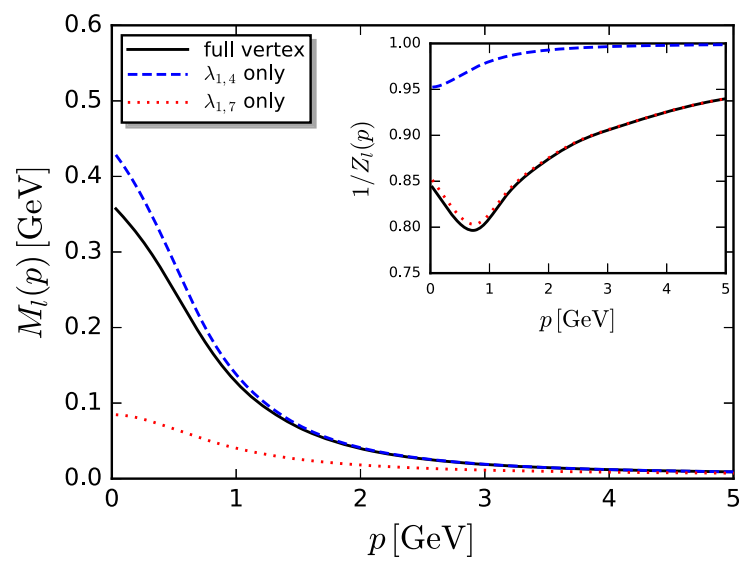

(b)

FIG. 12. Lack of quantitative reliability without quark-gluon couplings $\bar{\lambda}_{4,7}$ on the right-hand sides of the quark-gluon SDEs and the quark gap equation. (a) Quark dressings $M_{l}(p)$ and $1 / Z_{l}(p)$ without $\bar{\lambda}_{4}$ (dashed, red), and without $\bar{\lambda}_{7}$ (dotted, blue) in the vertex SDEs in comparison to the full solution (full, black). (b) Quark dressings $M_{l}(p)$ and $1 / Z_{l}(p)$ without $\bar{\lambda}_{4}$ (dashed, red), and without $\bar{\lambda}_{7}$ (dotted, blue) in the quark gap equation in comparison to the full solution (full, black).

contributions; at the corresponding peaks, $\bar{\lambda}_{1}>\bar{\lambda}_{7}>\left|\bar{\lambda}_{4}\right|$. In fact, the subleading form factors are even less relevant than suggested by the suppression of the couplings.

Importantly, the above study implies that the sole use of STI-derived vertices (which, by construction, do not include $\lambda_{4,7}$ ) in either the quark-gluon SDE or the gap equation leads to loss of quantitative precision. In particular, we have checked that the inclusion of the BC tensor structures alone in the gap equation reduces dramatically the amount of chiral symmetry breaking, yielding $M_{l}(0)<50 \mathrm{MeV}$.

For the evaluation of point (ii), we have computed $M_{q}(p)$ and $1 / Z_{q}(p)$ with a dressing $\lambda_{1}(p)$ obtained from the STI construction, while for $\lambda_{4,7}(p)$ we resort to scaling relations suggested by the underlying gauge-invariant tensor structures $[60,61,81]$. The results obtained are in

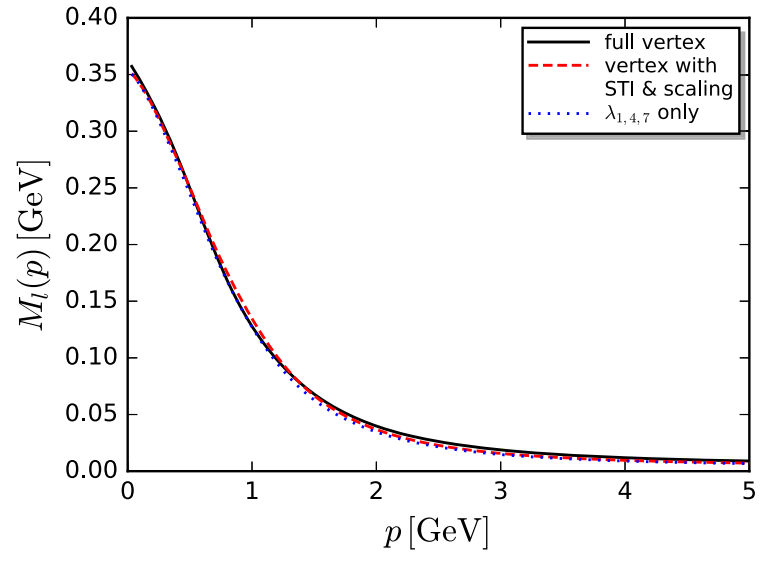

(a) excellent agreement with those of the full computation, as can be seen in Fig. 13.

The above analysis supports the appealing possibility of implementing relatively simple but quantitatively reliable approximations for hadron resonance computations or the phase structure of QCD (see also [79]); such a setup is currently under investigation.

\section{SUMMARY}

In this work we have considered the full set of SDEs describing the quark sector of $2+1$-flavor QCD. In particular, we have coupled the gap equation of the quark propagator with the one-loop dressed SDE of the quarkgluon vertex, and solved the resulting system of integral

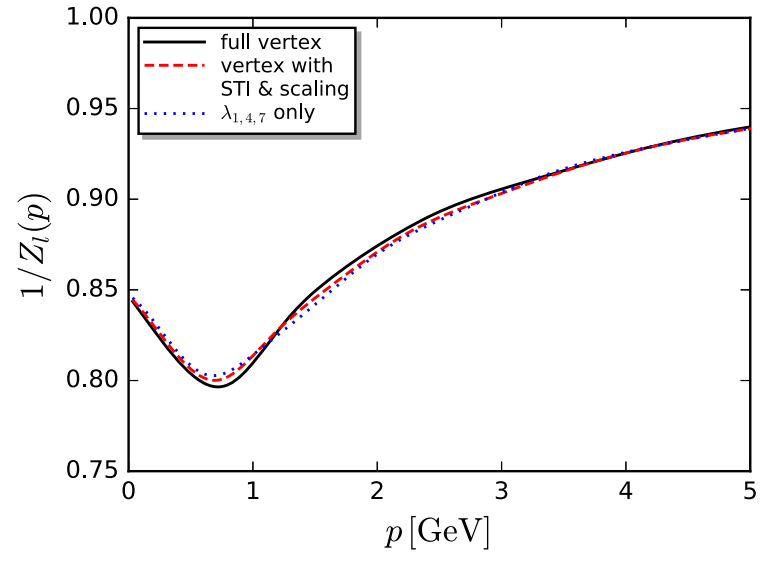

(b)

FIG. 13. Quantitative reliability of approximations: (a) Quark-gluon couplings $\lambda_{1,2,6}$ from STI, and $\lambda_{4,5,6,7}$ from scaling relations; see text and $[60,81]$. (b) $\lambda_{1,4,7}$. 
equations iteratively. The sole external ingredient used in this analysis is the gluon propagator, which has been taken from the lattice simulations of $[31,83,84]$ and results obtained from previous functional treatments $[58,60,61]$. Note, in particular, that the gauge coupling has been determined self-consistently, capturing correctly the analytic two-loop running.

The results of our analysis agree quantitatively with those of $N_{f}=2+1$ lattice simulations [106], and the combined (fRG and SDE) functional approach of [60,61]. In fact, our agreement with these latter approaches constitutes an important consistency check within functional methods: SDEs and fRG represent similar but distinct nonperturbative frameworks, and the coincidence of the respective results is highly nontrivial. Moreover, the value for the chiral condensate, our benchmark observable, has been compared to recent lattice predictions compiled in the FLAG review [99], showing excellent agreement (see Sec. VI B).

Finally, we have established that the form factors $\lambda_{1,4,7}(p)$ provide the dominant numerical contribution to the chiral infrared dynamics, as already suggested by previous studies $[60,61,81]$. This allows us to devise simplified but quantitatively reliable approximations, which may reduce the numerical costs in the study of systems governed by a large number of intertwined dynamical equations.

In summary, the results of the current comprehensive SDE approach provide physics results without the need of phenomenological infrared parameters that are commonly used explicitly or implicitly. Moreover, we have shown how to self-consistently incorporate in our analysis general external inputs.

In our opinion, the present comprehensive SDE approach, and in particular the combined use of functional relations for correlation functions, is essential for a successful quantitative investigation of many open physics problems in QCD, ranging from the hadron boundstate properties to the chiral phase structure and critical end point.

\section{ACKNOWLEDGMENTS}

We thank A. C. Aguilar, C. F. Fischer, M. Q. Huber, and G. Eichmann for discussions. F. G. is supported by the Alexander von Humboldt foundation. This work is supported by EMMI and the BMBF Grant No. 05P18VHFCA, by the Spanish Ministry of Economy and Competitiveness (MINECO) under Grant No. FPA2017-84543-P, and the Grant No. Prometeo/2019/087 of the Generalitat Valenciana. It is part of and supported by the DFG Collaborative Research Centre SFB 1225 (ISOQUANT) and the DFG under Germany's Excellence Strategy EXC2181/1-390900948 (the Heidelberg Excellence Cluster STRUCTURES).

\section{APPENDIX A: RENORMALIZATION SCHEME}

In this appendix we present additional details related to the renormalization scheme adapted in the present work.

\section{Mapping RG schemes}

In the fRG approach, for $k \rightarrow \infty$, all quantum fluctuations are suppressed, and the effective action $\Gamma_{k}$ of the fRG approach tends toward the bare action of QCD. This also entails that the momentum dependence of the dressings of correlation functions is subleading and drops with powers of $p^{2} / k^{2}$ : the dressings tend toward the renormalization factors of the bare action, within a momentum-cutoff renormalization. Accordingly, the $k$ dependence of effective action in the fRG approach translates to a dependence on the UV cutoff, $\Lambda$ in the present SDE approach. Moreover, the dependence on the renormalization scale $\mu$ is the same. In particular, logarithmically divergent RG factors run with $\log \Lambda^{2} / k_{\text {ref }}^{2}$, where $k_{\text {ref }}$ denotes some reference scale, typically large. This logarithmic $\Lambda$ dependence precisely cancels that produced by the integrated flows, where the cutoff integration runs from $k=\Lambda \rightarrow k=0$. This integrated flow agrees with the regularized SDE diagrams (again with UV cutoff $\Lambda$ ). Naturally, there is a very specific choice of $k_{\text {ref }}$, namely $k_{\text {ref }}=\Lambda$. Then, the logarithmic term vanishes, and we can put all $Z_{\phi_{i}, k=\Lambda}=1, M_{q, \Lambda}=m_{q}$, and $\lambda_{A^{3}, \Lambda}=\lambda_{c \bar{c} A, \Lambda}=\lambda_{q \bar{q} A, \Lambda}=g_{s}, \lambda_{A^{4}, \Lambda}=g_{s}^{2}$. This amounts to mapping the (implicit) RG scheme within the fRG to a standard RG scheme in the SDEs. In practice, this has to be accompanied with setting the RG scale to $\mu^{2}=\Lambda^{2}$, and finally removing the cutoff scale $k$.

In turn, for $k \rightarrow 0$, the fRG dressings are simply the finite dressings of the full, renormalized theory, and no dependence on the cutoff scale is left. This property is called $R G$ consistency; see $[8,108,109]$.

\section{One- and two-loop $\alpha_{s}(p)$}

In Sec. VII, the full quark-gluon coupling has been compared with its one- and two-loop counterparts (see in particular Fig. 7). It is here, where the difference between the current fRG-based MOM-type scheme, described in Secs. VA and A 1, and the standard MOM scheme becomes most apparent.

For the comparison with the one-loop $\alpha_{s}$, we use the standard parametrization of the latter, e.g., [110],

$$
\begin{aligned}
\alpha_{s}^{(1 \text { loop })}(p) & =\frac{1}{\beta_{0} \ln \left(p^{2} / \Lambda_{\mathrm{QCD}}^{2}\right)}, \\
\Lambda_{\mathrm{QCD}}^{2} & =\mu^{2} \exp \left[-\frac{1}{\beta_{0} \alpha_{s}(\mu)}\right],
\end{aligned}
$$

with $\beta_{0}=\frac{1}{4 \pi}\left(11-\frac{2}{3} N_{f}\right)$. As is clear from (A1), $\Lambda_{\mathrm{QCD}}$ provides the position of the singularity of $\alpha_{s}^{(1 \mathrm{loop})}(p)$ in momentum space, $p_{\text {sing }}:=\Lambda_{\mathrm{QCD}}$. This definition is also 
used below for the two-loop coupling, $\alpha_{s}^{(2 \text { loop })}(p)$. We also emphasize that while being natural, it is not the only possible definition of $\Lambda_{\mathrm{QCD}}$ beyond one loop; see, e.g., [111].

Even though $\Lambda_{\mathrm{QCD}}$ is, in principle, $\mu$ independent, this property is not exhibited at the level of the one-loop formulas. Specifically, using the two combinations of $\left\{\mu, \alpha_{s}(\mu)\right\}$ given in (5.5), we obtain $\Lambda_{\mathrm{QCD}}=0.59 \mathrm{GeV}$ for $\mu=40 \mathrm{GeV}$, and $\Lambda_{\mathrm{QCD}}=0.86 \mathrm{GeV}$ for $\mu=4.3 \mathrm{GeV}$. The large difference between the respective $\Lambda_{\mathrm{QCD}}$ is yet another manifestation of the limitations of the one-loop approximation. These limitations prevent us from using large RG scales even in the context of the standard MOM scheme. Moreover, the analysis also entails that $\Lambda_{\mathrm{QCD}}$ in the current MOM-type scheme is significantly different from the respective value in the standard MOM scheme. This has already been discussed in detail in [112], where a comparison of the present scheme with the $\overline{\mathrm{MS}}$ scheme was carried out within Yang-Mills theory. This analysis extends straightforwardly to a comparison with MOM. The difference with the standard MOM scheme is also clearly seen in the comparison of the Yang-Mills data of [96] (fRG, present scheme) with those of [88] (SDE, MOM scheme), both featuring correlation functions in quantitative agreement with the respective lattice results.

For the two loop comparison we use the standard parametrization given, e.g., in [110],

$\alpha_{s}^{(2 \text { loop })}(p)=-\frac{\beta_{0}}{\beta_{1}} \frac{1}{1+W_{-1}(z)}, \quad z=-\frac{\beta_{0}^{2}}{e \beta_{1}}\left(\frac{\Lambda_{\mathrm{QCD}}^{2}}{p^{2}}\right)^{\beta_{0}^{2} / \beta_{1}}$,

with $\beta_{1}=\frac{1}{(4 \pi)^{2}}\left(102-\frac{38}{3} N_{f}\right)$ and $\beta_{0}=\frac{1}{4 \pi}\left(11-\frac{2}{3} N_{f}\right)$, where $W_{-1}(z)$ denotes the "physical" branch of the real valued Lambert function.

We also note in passing that the approximate formula [113]

$$
\alpha_{s}^{(2 \text { loop })}(p)=\frac{\alpha(\mu)}{1+\beta_{0} \alpha(\mu)\left[1+\alpha(\mu) \frac{\beta_{1}}{\beta_{0}}\right] \ln \left(p^{2} / \mu^{2}\right)}
$$

fits the full coupling $\alpha_{\overline{l l} A}(p)$ even better than (A2) for momenta $p \gtrsim 10 \mathrm{GeV}$ (in terms of $\chi^{2}$ ). This may be interpreted as an indication of the effectiveness of the resummation scheme underlying our approximation in the perturbative regime.

As is clear from Fig. 14, $p_{\text {sing }}=\Lambda_{\mathrm{QCD}}$ of $\alpha_{s}^{(2 \text { loop })}(p)$ is stable under changes of $\mu$ for $\mu \gtrsim 10 \mathrm{GeV}$ and is still compatible in a transition regime with $p \in(5-10) \mathrm{GeV}$. This is in clear contradistinction to its one-loop counterpart, where such an RG consistency does not hold. In particular, we find that $\Lambda_{\mathrm{QCD}}=1.42 \mathrm{GeV}$ for $\mu=40 \mathrm{GeV}$, and $\Lambda_{\mathrm{QCD}}=1.49 \mathrm{GeV}$ for our low RG scale

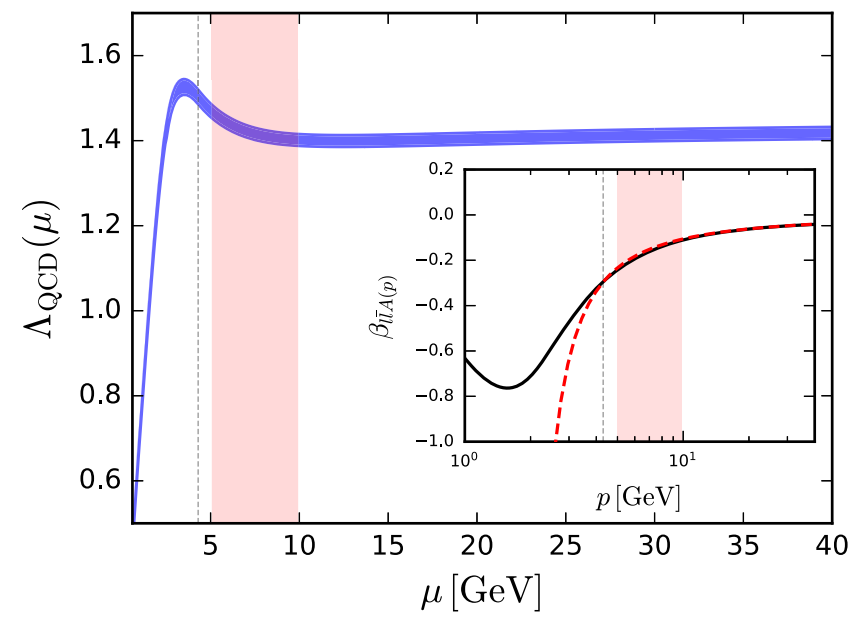

FIG. 14. $\Lambda_{\mathrm{QCD}}(\mu)=p_{\text {sing }}$, defined by the singularity of $\alpha_{s}^{(2 \text { loop })}(p)$, for $\mu \in(1,40) \mathrm{GeV}$. The RG scale $\mu=4.3 \mathrm{GeV}$ is indicated with a dashed vertical line. The inset shows the full $\beta$-function, $\beta_{\overline{l l} A}\left(\alpha_{S}\right)$, in comparison to its two-loop counterpart. The red vertical band indicates the transition momentum regime, in which the full $\beta$-function starts to deviate from the perturbative two-loop $\beta$-function.

of $\mu=4.3 \mathrm{GeV}$. The latter RG scale is at the limit or slightly below the lower bound of the two-loop consistent regime. Note that this low RG scale has been chosen because it represents the largest momentum accessible by the lattice data.

To sum up, the large $\Lambda_{\mathrm{QCD}}$ values compared to those of the standard MOM are expected from respective comparisons in Yang-Mills theory, as discussed in this appendix. Moreover, the $\mu$ dependence for $\mu \lesssim 10 \mathrm{GeV}$ within a twoloop matching is expected, given that the $\beta$-function and hence the coupling deviate from their two-loop counterparts in the transition regime $(5-10 \mathrm{GeV})$ indicated by the vertical red band; see Fig. 7 and the inset of Fig. 14.

\section{APPENDIX B: KERNELS OF THE QUARK-GLUON VERTEX SDE}

The kernels $K_{i j k}(p, q, k)$ and $\tilde{K}_{i j k}(p, q, k)$ appearing in (4.8) have the general form

$$
\begin{aligned}
& K_{i j k}(p, q, k)=\sum_{\alpha=1}^{2} C_{i j k}^{\alpha}(p, q, k) \sigma_{\alpha}(k), \\
& \tilde{K}_{i j k}(p, q, k)=\sum_{\alpha=1}^{4} \tilde{C}_{i j k}^{\alpha}(p, q, k) \tilde{\sigma}_{\alpha}(p, q, k),
\end{aligned}
$$

with

$\sigma_{1}(k)=\frac{1}{Z_{q}(k)\left[k^{2}+M_{q}^{2}(k)\right]}, \quad \sigma_{2}(k)=\frac{M_{q}(k)}{Z_{q}(k)\left[k^{2}+M_{q}^{2}(k)\right]}$, 
and

$$
\begin{aligned}
& \tilde{\sigma}_{1}(p, q, k)=\frac{1}{R(p, q, k)}, \quad \tilde{\sigma}_{2}(p, q, k)=\frac{M_{q}(q+k)}{R(p, q, k)}, \\
& \tilde{\sigma}_{3}(p, q, k)=\frac{M_{q}(p+k)}{R(p, q, k)}, \\
& \tilde{\sigma}_{4}(p, q, k)=\frac{M_{q}(q+k) M_{q}(p+k)}{R(p, q, k)},
\end{aligned}
$$

where

$$
\begin{aligned}
R(p, q, k):= & Z_{q}(p+k) Z_{q}(q+k)\left[(p+k)^{2}\right. \\
& \left.+M_{q}^{2}(p+k)\right]\left[(q+k)^{2}+M_{q}^{2}(q+k)\right],
\end{aligned}
$$

and the closed expressions for the kinematic functions $C_{i j k}^{\alpha}(p, q, k)$ and $\tilde{C}_{i j k}^{\alpha}(p, q, k)$ are reported in the github (https://github.com/coupledSDE/FormDerive).
[1] C. D. Roberts and A. G. Williams, Prog. Part. Nucl. Phys. 33, 477 (1994).

[2] R. Alkofer and L. von Smekal, Phys. Rep. 353, 281 (2001).

[3] P. Maris and C. D. Roberts, Int. J. Mod. Phys. E 12, 297 (2003).

[4] C. S. Fischer, J. Phys. G 32, R253 (2006).

[5] D. Binosi and J. Papavassiliou, Phys. Rep. 479, 1 (2009).

[6] A. Maas, Phys. Rep. 524, 203 (2013).

[7] M. Q. Huber, Phys. Rep. 879, 1 (2020).

[8] J. M. Pawlowski, Ann. Phys. (Amsterdam) 322, 2831 (2007).

[9] H. Gies, Lect. Notes Phys. 852, 287 (2012).

[10] O. J. Rosten, Phys. Rep. 511, 177 (2012).

[11] J. Braun, J. Phys. G 39, 033001 (2012).

[12] J. M. Pawlowski, Nucl. Phys. 931, 113 (2014).

[13] N. Dupuis, L. Canet, A. Eichhorn, W. Metzner, J. M. Pawlowski, M. Tissier, and N. Wschebor, Phys. Rep. (2020).

[14] I. C. Cloet and C. D. Roberts, Prog. Part. Nucl. Phys. 77, 1 (2014).

[15] G. Eichmann, H. Sanchis-Alepuz, R. Williams, R. Alkofer, and C. S. Fischer, Prog. Part. Nucl. Phys. 91, 1 (2016).

[16] H. Sanchis-Alepuz and R. Williams, Comput. Phys. Commun. 232, 1 (2018).

[17] A. Cucchieri and T. Mendes, Proc. Sci., LATTICE2007 (2007) 297.

[18] I. L. Bogolubsky, E. M. Ilgenfritz, M. Muller-Preussker, and A. Sternbeck, Proc. Sci., LATTICE2007 (2007) 290.

[19] P. O. Bowman, U. M. Heller, D. B. Leinweber, M. B. Parappilly, A. Sternbeck, L. von Smekal, A. G. Williams, and J.-B. Zhang, Phys. Rev. D 76, 094505 (2007).

[20] I. L. Bogolubsky, E. M. Ilgenfritz, M. Muller-Preussker, and A. Sternbeck, Phys. Lett. B 676, 69 (2009).

[21] O. Oliveira and P. J. Silva, Proc. Sci., LAT2009 (2009) 226.

[22] J. Skullerud and A. Kizilersu, J. High Energy Phys. 09 (2002) 013.

[23] J. I. Skullerud, P. O. Bowman, A. Kizilersu, D. B. Leinweber, and A. G. Williams, J. High Energy Phys. 04 (2003) 047.

[24] A. Kizilersu, D. B. Leinweber, J.-I. Skullerud, and A. G. Williams, Eur. Phys. J. C 50, 871 (2007).
[25] E. Rojas, J. P. B. C. de Melo, B. El-Bennich, O. Oliveira, and T. Frederico, J. High Energy Phys. 10 (2013) 193.

[26] O. Oliveira, A. Kızılersu, P. J. Silva, J.-I. Skullerud, A. Sternbeck, and A. G. Williams, Acta Phys. Pol. B Proc. Suppl. 9, 363 (2016).

[27] A. Sternbeck, P.-H. Balduf, A. Kızılersu, O. Oliveira, P. J. Silva, J.-I. Skullerud, and A. G. Williams, Proc. Sci., LATTICE2016 (2017) 349.

[28] O. Oliveira, T. Frederico, W. de Paula, and J. P. B. C. de Melo, Eur. Phys. J. C 78, 553 (2018).

[29] A. Athenodorou, D. Binosi, P. Boucaud, F. De Soto, J. Papavassiliou, J. Rodriguez-Quintero, and S. Zafeiropoulos, Phys. Lett. B 761, 444 (2016).

[30] A. G. Duarte, O. Oliveira, and P. J. Silva, Phys. Rev. D 94, 074502 (2016).

[31] A. C. Aguilar, F. De Soto, M. N. Ferreira, J. Papavassiliou, J. Rodríguez-Quintero, and S. Zafeiropoulos, Eur. Phys. J. C 80, 154 (2020).

[32] A. C. Aguilar, F. De Soto, M. N. Ferreira, J. Papavassiliou, and J. Rodríguez-Quintero, arXiv:2102.04959.

[33] A. Bender, W. Detmold, C. D. Roberts, and A. W. Thomas, Phys. Rev. C 65, 065203 (2002).

[34] R. Alkofer, C. S. Fischer, F. J. Llanes-Estrada, and K. Schwenzer, Ann. Phys. (Amsterdam) 324, 106 (2009).

[35] L. Chang and C. D. Roberts, Phys. Rev. Lett. 103, 081601 (2009).

[36] A. C. Aguilar and J. Papavassiliou, Phys. Rev. D 83, 014013 (2011).

[37] G. Eichmann, Phys. Rev. D 84, 014014 (2011).

[38] D. Binosi, L. Chang, J. Papavassiliou, and C. D. Roberts, Phys. Lett. B 742, 183 (2015).

[39] M. Gómez-Rocha, T. Hilger, and A. Krassnigg, Few Body Syst. 56, 475 (2015).

[40] M. Gomez-Rocha, T. Hilger, and A. Krassnigg, Phys. Rev. D 92, 054030 (2015).

[41] G. Eichmann, C. S. Fischer, and H. Sanchis-Alepuz, Phys. Rev. D 94, 094033 (2016).

[42] D. Binosi, L. Chang, J. Papavassiliou, S.-X. Qin, and C. D. Roberts, Phys. Rev. D 93, 096010 (2016).

[43] J. Braun, H. Gies, and J. M. Pawlowski, Phys. Lett. B 684, 262 (2010).

[44] J. Braun, L. M. Haas, F. Marhauser, and J. M. Pawlowski, Phys. Rev. Lett. 106, 022002 (2011). 
[45] S.-X. Qin, L. Chang, H. Chen, Y.-X. Liu, and C. D. Roberts, Phys. Rev. Lett. 106, 172301 (2011).

[46] C. S. Fischer, J. Luecker, and J. A. Mueller, Phys. Lett. B 702, 438 (2011).

[47] L.-J. Luo, S. Shi, and H.-S. Zong, Mod. Phys. Lett. A 28, 1350105 (2013).

[48] L. Fister and J. M. Pawlowski, Phys. Rev. D 88, 045010 (2013).

[49] C. S. Fischer, L. Fister, J. Luecker, and J. M. Pawlowski, Phys. Lett. B 732, 273 (2014).

[50] C. S. Fischer, J. Luecker, and C. A. Welzbacher, Phys. Rev. D 90, 034022 (2014).

[51] N. Christiansen, M. Haas, J. M. Pawlowski, and N. Strodthoff, Phys. Rev. Lett. 115, 112002 (2015).

[52] C. Shi, Y.-L. Wang, Y. Jiang, Z.-F. Cui, and H.-S. Zong, J. High Energy Phys. 07 (2014) 014.

[53] Z.-F. Cui, F.-Y. Hou, Y.-M. Shi, Y.-L. Wang, and H.-S. Zong, Ann. Phys. (Amsterdam) 358, 172 (2015).

[54] G. Eichmann, C. S. Fischer, and C. A. Welzbacher, Phys. Rev. D 93, 034013 (2016).

[55] A. K. Cyrol, M. Mitter, J. M. Pawlowski, and N. Strodthoff, Phys. Rev. D 97, 054015 (2018).

[56] R. Contant, M. Q. Huber, C. S. Fischer, C. A. Welzbacher, and R. Williams, Acta Phys. Pol. B Proc. Suppl. 11, 483 (2018).

[57] J. Maelger, U. Reinosa, and J. Serreau, Phys. Rev. D 101, 014028 (2020).

[58] W.-J. Fu, J. M. Pawlowski, and F. Rennecke, Phys. Rev. D 101, 054032 (2020).

[59] J. Braun, M. Leonhardt, and M. Pospiech, Phys. Rev. D 101, 036004 (2020).

[60] F. Gao and J. M. Pawlowski, Phys. Rev. D 102, 034027 (2020).

[61] F. Gao and J. M. Pawlowski, arXiv:2010.13705.

[62] J. Braun, W.-J. Fu, J. M. Pawlowski, F. Rennecke, D. Rosenblüh, and S. Yin, Phys. Rev. D 102, 056010 (2020).

[63] J. S. Ball and T.-W. Chiu, Phys. Rev. D 22, 2542 (1980).

[64] D. C. Curtis and M. R. Pennington, Phys. Rev. D 42, 4165 (1990).

[65] A. I. Davydychev, P. Osland, and L. Saks, Phys. Rev. D 63, 014022 (2000).

[66] C. S. Fischer and R. Alkofer, Phys. Rev. D 67, 094020 (2003).

[67] A. C. Aguilar, D. Binosi, D. Ibañez, and J. Papavassiliou, Phys. Rev. D 90, 065027 (2014).

[68] A. C. Aguilar, J. C. Cardona, M. N. Ferreira, and J. Papavassiliou, Phys. Rev. D 96, 014029 (2017).

[69] R. Bermudez, L. Albino, L. X. Gutiérrez-Guerrero, M. E. Tejeda-Yeomans, and A. Bashir, Phys. Rev. D 95, 034041 (2017).

[70] A. C. Aguilar, J. C. Cardona, M. N. Ferreira, and J. Papavassiliou, Phys. Rev. D 98, 014002 (2018).

[71] M. Peláez, U. Reinosa, J. Serreau, M. Tissier, and N. Wschebor, Phys. Rev. D 96, 114011 (2017).

[72] M. Peláez, U. Reinosa, J. Serreau, M. Tissier, and N. Wschebor, arXiv:2010.13689.

[73] N. Barrios, J. A. Gracey, M. Peláez, and U. Reinosa, arXiv:2103.16218.

[74] C. S. Fischer and R. Williams, Phys. Rev. Lett. 103, 122001 (2009).
[75] L. Chang, Y.-X. Liu, and C. D. Roberts, Phys. Rev. Lett. 106, 072001 (2011).

[76] R. Williams, Eur. Phys. J. A 51, 57 (2015).

[77] M. Mitter, J. M. Pawlowski, and N. Strodthoff, Phys. Rev. D 91, 054035 (2015).

[78] A. L. Blum, R. Alkofer, M. Q. Huber, and A. Windisch, Acta Phys. Pol. B Proc. Suppl. 8, 321 (2015).

[79] R. Williams, C. S. Fischer, and W. Heupel, Phys. Rev. D 93, 034026 (2016).

[80] D. Binosi, L. Chang, J. Papavassiliou, S.-X. Qin, and C. D. Roberts, Phys. Rev. D 95, 031501 (2017).

[81] A. K. Cyrol, M. Mitter, J. M. Pawlowski, and N. Strodthoff, Phys. Rev. D 97, 054006 (2018).

[82] C. Tang, F. Gao, and Y.-X. Liu, Phys. Rev. D 100, 056001 (2019).

[83] P. Boucaud, F. De Soto, K. Raya, J. RodríguezQuintero, and S. Zafeiropoulos, Phys. Rev. D 98, 114515 (2018).

[84] S. Zafeiropoulos, P. Boucaud, F. De Soto, J. RodríguezQuintero, and J. Segovia, Phys. Rev. Lett. 122, 162002 (2019).

[85] A. Ayala, A. Bashir, D. Binosi, M. Cristoforetti, and J. Rodriguez-Quintero, Phys. Rev. D 86, 074512 (2012).

[86] J. C. R. Bloch, Phys. Rev. D 64, 116011 (2001).

[87] J. C. R. Bloch, Phys. Rev. D 66, 034032 (2002).

[88] M. Q. Huber, Phys. Rev. D 101, 114009 (2020).

[89] A. K. Cyrol, M. Q. Huber, and L. von Smekal, Eur. Phys. J. C 75, 102 (2015).

[90] M. Q. Huber, Phys. Rev. D 93, 085033 (2016).

[91] M. Q. Huber, Eur. Phys. J. C 77, 733 (2017).

[92] A. C. Aguilar, D. Binosi, and J. Papavassiliou, Phys. Rev. D 78, 025010 (2008).

[93] A. C. Aguilar, D. Binosi, and J. Papavassiliou, Phys. Rev. D 86, 014032 (2012).

[94] P. Boucaud, J. P. Leroy, A. Le Yaouanc, J. Micheli, O. Pene, and J. Rodriguez-Quintero, J. High Energy Phys. 06 (2008) 099.

[95] C. S. Fischer, A. Maas, and J. M. Pawlowski, Ann. Phys. (Amsterdam) 324, 2408 (2009).

[96] A. K. Cyrol, L. Fister, M. Mitter, J. M. Pawlowski, and N. Strodthoff, Phys. Rev. D 94, 054005 (2016).

[97] A. C. Aguilar, D. Ibanez, V. Mathieu, and J. Papavassiliou, Phys. Rev. D 85, 014018 (2012).

[98] A. C. Aguilar, D. Binosi, C. T. Figueiredo, and J. Papavassiliou, Phys. Rev. D 94, 045002 (2016).

[99] S. Aoki et al. (Flavour Lattice Averaging Group) Eur. Phys. J. C 80, 113 (2020).

[100] C. S. Fischer, Prog. Part. Nucl. Phys. 105, 1 (2019).

[101] A. Bender, G. I. Poulis, C. D. Roberts, S. M. Schmidt, and A. W. Thomas, Phys. Lett. B 431, 263 (1998).

[102] F. Gao and Y.-X. Liu, Phys. Rev. D 97, 056011 (2018).

[103] A. Bashir, L. Chang, I. C. Cloet, B. El-Bennich, Y.-X. Liu, C. D. Roberts, and P. C. Tandy, Commun. Theor. Phys. 58, 79 (2012).

[104] P. Maris, C. D. Roberts, and P. C. Tandy, Phys. Lett. B 420, 267 (1998).

[105] V. Miransky, Dynamical Symmetry Breaking in Quantum Field Theories (WSPC, Singapore, 1994). 
[106] P. O. Bowman, U. M. Heller, D. B. Leinweber, M. B. Parappilly, A. G. Williams, and J.-B. Zhang, Phys. Rev. D 71, 054507 (2005).

[107] L. Corell, A. K. Cyrol, M. Mitter, J. M. Pawlowski, and N. Strodthoff, SciPost Phys. 5, 066 (2018).

[108] J. M. Pawlowski, M. M. Scherer, R. Schmidt, and S. J. Wetzel, Ann. Phys. (Amsterdam) 384, 165 (2017).
[109] J. Braun, M. Leonhardt, and J. M. Pawlowski, SciPost Phys. 6, 056 (2019).

[110] G. M. Prosperi, M. Raciti, and C. Simolo, Prog. Part. Nucl. Phys. 58, 387 (2007).

[111] A. Deur, S. J. Brodsky, and G. F. de Teramond, Nucl. Phys. 90, 1 (2016).

[112] U. Ellwanger, Z. Phys. C 76, 721 (1997).

[113] A. Pich, Prog. Part. Nucl. Phys. 117, 103846 (2021). 